DESY-06-128

August 2006

\title{
Inclusive-jet and dijet cross sections in deep inelastic scattering at HERA
}

\author{
ZEUS Collaboration
}

\begin{abstract}
Inclusive-jet and dijet differential cross sections have been measured in neutral current deep inelastic ep scattering for exchanged boson virtualities $Q^{2}>$ $125 \mathrm{GeV}^{2}$ with the ZEUS detector at HERA using an integrated luminosity of $82 \mathrm{pb}^{-1}$. Jets were identified in the Breit frame using the $k_{T}$ cluster algorithm. Jet cross sections are presented as functions of several kinematic and jet variables. The results are also presented in different regions of $Q^{2}$. Next-to-leadingorder QCD calculations describe the measurements well. Regions of phase space where the theoretical uncertainties are small have been identified. Measurements in these regions have the potential to constrain the gluon density in the proton when used as inputs to global fits of the proton parton distribution functions.
\end{abstract}




\section{The ZEUS Collaboration}

S. Chekanov, M. Derrick, S. Magill, S. Miglioranzi ${ }^{1}$, B. Musgrave, D. Nicholass ${ }^{1}$, J. Repond, R. Yoshida Argonne National Laboratory, Argonne, Illinois 60439-4815, USA ${ }^{n}$

M.C.K. Mattingly

Andrews University, Berrien Springs, Michigan 49104-0380, USA

N. Pavel ${ }^{\dagger}$, A.G. Yagües Molina

Institut für Physik der Humboldt-Universität zu Berlin, Berlin, Germany

S. Antonelli, P. Antonioli, G. Bari, M. Basile, L. Bellagamba, M. Bindi, D. Boscherini, A. Bruni, G. Bruni, L. Cifarelli, F. Cindolo, A. Contin, M. Corradi ${ }^{2}$, S. De Pasquale, G. Iacobucci, A. Margotti, R. Nania, A. Polini, L. Rinaldi, G. Sartorelli, A. Zichichi University and INFN Bologna, Bologna, Italy ${ }^{e}$

G. Aghuzumtsyan, D. Bartsch, I. Brock, S. Goers, H. Hartmann, E. Hilger, H.-P. Jakob, M. Jüngst, O.M. Kind, E. Paul ${ }^{3}$, J. Rautenberg ${ }^{4}$, R. Renner, U. Samson ${ }^{5}$, V. Schönberg, M. Wang, M. Wlasenko

Physikalisches Institut der Universität Bonn, Bonn, Germany ${ }^{b}$

N.H. Brook, G.P. Heath, J.D. Morris, T. Namsoo

H.H. Wills Physics Laboratory, University of Bristol, Bristol, United Kingdom ${ }^{m}$

M. Capua, S. Fazio, A. Mastroberardino, M. Schioppa, G. Susinno, E. Tassi

Calabria University, Physics Department and INFN, Cosenza, Italy ${ }^{e}$

J.Y. $\mathrm{Kim}^{6}$, K.J. $\mathrm{Ma}^{7}$

Chonnam National University, Kwang.ju, South Korea ${ }^{g}$

Z.A. Ibrahim, B. Kamaluddin, W.A.T. Wan Abdullah

Jabatan Fizik, Universiti Malaya, 50603 Kuala Lumpur, Malaysia ${ }^{r}$

Y. Ning, Z. Ren, F. Sciulli

Nevis Laboratories, Columbia University, Irvington on Hudson, New York 10027\%

J. Chwastowski, A. Eskreys, J. Figiel, A. Galas, M. Gil, K. Olkiewicz, P. Stopa, L. Zawiejski

The Henryk Niewodniczanski Institute of Nuclear Physics, Polish Academy of Sciences, Cracow, Poland ${ }^{i}$

L. Adamczyk, T. Bołd, I. Grabowska-Bołd, D. Kisielewska, J. Łukasik, M. Przybycień,

L. Suszycki

Faculty of Physics and Applied Computer Science, AGH-University of Science and Technology, Cracow, Poland ${ }^{p}$ 
A. Kotański ${ }^{8}$, W. Słomiński

Department of Physics, Jagellonian University, Cracow, Poland

V. Adler, U. Behrens, I. Bloch, A. Bonato, K. Borras, N. Coppola, J. Fourletova, A. Geiser,

D. Gladkov, P. Göttlicher ${ }^{9}$, I. Gregor, O. Gutsche, T. Haas, W. Hain, C. Horn, B. Kahle,

U. Kötz, H. Kowalski, H. Lim ${ }^{10}$, E. Lobodzinska, B. Löhr, R. Mankel, I.-A. Melzer-

Pellmann, A. Montanari, C.N. Nguyen, D. Notz, A.E. Nuncio-Quiroz, R. Santamarta,

U. Schneekloth, A. Spiridonov ${ }^{11}$, H. Stadie, U. Stösslein, D. Szuba ${ }^{12}$, J. Szuba ${ }^{13}$, T. Theedt,

G. Watt, G. Wolf, K. Wrona, C. Youngman, W. Zeuner

Deutsches Elektronen-Synchrotron DESY, Hamburg, Germany

S. Schlenstedt

Deutsches Elektronen-Synchrotron DESY, Zeuthen, Germany

G. Barbagli, E. Gallo, P. G. Pelfer

University and INFN, Florence, Italy ${ }^{e}$

A. Bamberger, D. Dobur, F. Karstens, N.N. Vlasov ${ }^{14}$

Fakultät für Physik der Universität Freiburg i.Br., Freiburg i.Br., Germany ${ }^{b}$

P.J. Bussey, A.T. Doyle, W. Dunne, J. Ferrando, D.H. Saxon, I.O. Skillicorn

Department of Physics and Astronomy, University of Glasgow, Glasgow, United Kingdom, ${ }^{m}$

I. Gialas ${ }^{15}$

Department of Engineering in Management and Finance, Univ. of Aegean, Greece

T. Gosau, U. Holm, R. Klanner, E. Lohrmann, H. Salehi, P. Schleper, T. Schörner-Sadenius, J. Sztuk, K. Wichmann, K. Wick

Hamburg University, Institute of Exp. Physics, Hamburg, Germany ${ }^{b}$

C. Foudas, C. Fry, K.R. Long, A.D. Tapper

Imperial College London, High Energy Nuclear Physics Group, London, United Kingdom $^{m}$

M. Kataoka ${ }^{16}$, T. Matsumoto, K. Nagano, K. Tokushuku ${ }^{17}$, S. Yamada, Y. Yamazaki Institute of Particle and Nuclear Studies, KEK, Tsukuba, Japan ${ }^{f}$

A.N. Barakbaev, E.G. Boos, A. Dossanov, N.S. Pokrovskiy, B.O. Zhautykov

Institute of Physics and Technology of Ministry of Education and Science of Kazakhstan, Almaty, Kazakhstan

D. Son

Kyungpook National University, Center for High Energy Physics, Daegu, South Korea ${ }^{g}$ 
J. de Favereau, K. Piotrzkowski

Institut de Physique Nucléaire, Université Catholique de Louvain, Louvain-la-Neuve, Belgium $^{q}$

F. Barreiro, C. Glasman ${ }^{18}$, M. Jimenez, L. Labarga, J. del Peso, E. Ron, J. Terrón, M. Zambrana

Departamento de Fúsica Teórica, Universidad Autónoma de Madrid, Madrid, Spain ${ }^{l}$

F. Corriveau, C. Liu, R. Walsh, C. Zhou

Department of Physics, McGill University, Montréal, Québec, Canada H3A 2T8 ${ }^{a}$

T. Tsurugai

Meiji Gakuin University, Faculty of General Education, Yokohama, Japan ${ }^{f}$

A. Antonov, B.A. Dolgoshein, I. Rubinsky, V. Sosnovtsev, A. Stifutkin, S. Suchkov Moscow Engineering Physics Institute, Moscow, Russia ${ }^{j}$

R.K. Dementiev, P.F. Ermolov, L.K. Gladilin, I.I. Katkov, L.A. Khein, I.A. Korzhavina, V.A. Kuzmin, B.B. Levchenko ${ }^{19}$, O.Yu. Lukina, A.S. Proskuryakov, L.M. Shcheglova, D.S. Zotkin, S.A. Zotkin

Moscow State University, Institute of Nuclear Physics, Moscow, Russia ${ }^{k}$

I. Abt, C. Büttner, A. Caldwell, D. Kollar, W.B. Schmidke, J. Sutiak

Max-Planck-Institut für Physik, München, Germany

G. Grigorescu, A. Keramidas, E. Koffeman, P. Kooijman, A. Pellegrino, H. Tiecke, M. Vázquez ${ }^{20}$, L. Wiggers

NIKHEF and University of Amsterdam, Amsterdam, Netherlands ${ }^{h}$

N. Brümmer, B. Bylsma, L.S. Durkin, A. Lee, T.Y. Ling

Physics Department, Ohio State University, Columbus, Ohio $43210^{n}$

P.D. Allfrey, M.A. Bell, A.M. Cooper-Sarkar, A. Cottrell, R.C.E. Devenish, B. Foster, C. Gwenlan ${ }^{21}$, K. Korcsak-Gorzo, S. Patel, V. Roberfroid ${ }^{22}$, A. Robertson, P.B. Straub, C. Uribe-Estrada, R. Walczak

Department of Physics, University of Oxford, Oxford United Kingdom ${ }^{m}$

P. Bellan, A. Bertolin, R. Brugnera, R. Carlin, R. Ciesielski, F. Dal Corso, S. Dusini,

A. Garfagnini, S. Limentani, A. Longhin, L. Stanco, M. Turcato

Dipartimento di Fisica dell' Università and INFN, Padova, Italy ${ }^{e}$

B.Y. Oh, A. Raval, J. Ukleja ${ }^{23}$, J.J. Whitmore

Department of Physics, Pennsylvania State University, University Park, Pennsylvania $16802^{\circ}$

Y. Iga

Polytechnic University, Sagamihara, Japan ${ }^{f}$ 
G. D'Agostini, G. Marini, A. Nigro

Dipartimento di Fisica, Università 'La Sapienza' and INFN, Rome, Italy ${ }^{e}$

J.E. Cole, J.C. Hart

Rutherford Appleton Laboratory, Chilton, Didcot, Oxon, United Kingdom ${ }^{m}$

H. Abramowicz ${ }^{24}$, A. Gabareen, R. Ingbir, S. Kananov, A. Levy

Raymond and Beverly Sackler Faculty of Exact Sciences, School of Physics, Tel-Aviv

University, Tel-Aviv, Israel ${ }^{d}$

M. Kuze

Department of Physics, Tokyo Institute of Technology, Tokyo, Japan ${ }^{f}$

R. Hori, S. Kagawa ${ }^{25}$, S. Shimizu, T. Tawara

Department of Physics, University of Tokyo, Tokyo, Japan ${ }^{f}$

R. Hamatsu, H. Kaji, S. Kitamura ${ }^{26}$, O. Ota, Y.D. Ri

Tokyo Metropolitan University, Department of Physics, Tokyo, Japan ${ }^{f}$

M.I. Ferrero, V. Monaco, R. Sacchi, A. Solano

Università di Torino and INFN, Torino, Italy ${ }^{e}$

M. Arneodo, M. Ruspa

Università del Piemonte Orientale, Novara, and INFN, Torino, Italy ${ }^{e}$

S. Fourletov, J.F. Martin

Department of Physics, University of Toronto, Toronto, Ontario, Canada M5S 1A7 a

S.K. Boutle ${ }^{15}$, J.M. Butterworth, R. Hall-Wilton ${ }^{20}$, T.W. Jones, J.H. Loizides, M.R. Sutton ${ }^{27}$, C. Targett-Adams, M. Wing

Physics and Astronomy Department, University College London, London, United Kingdom $^{m}$

B. Brzozowska, J. Ciborowski ${ }^{28}$, G. Grzelak, P. Kulinski, P. Łużniak ${ }^{29}$, J. Malka ${ }^{29}$, R.J. Nowak, J.M. Pawlak, T. Tymieniecka, A. Ukleja ${ }^{30}$, A.F. Żarnecki

Warsaw University, Institute of Experimental Physics, Warsaw, Poland

M. Adamus, P. Plucinski ${ }^{31}$

Institute for Nuclear Studies, Warsaw, Poland

Y. Eisenberg, I. Giller, D. Hochman, U. Karshon, M. Rosin

Department of Particle Physics, Weizmann Institute, Rehovot, Israel ${ }^{c}$

E. Brownson, T. Danielson, A. Everett, D. Kçira, D.D. Reeder, P. Ryan, A.A. Savin,

W.H. Smith, H. Wolfe

Department of Physics, University of Wisconsin, Madison, Wisconsin 53706, USA ${ }^{n}$ 
S. Bhadra, C.D. Catterall, Y. Cui, G. Hartner, S. Menary, U. Noor, M. Soares, J. Standage, J. Whyte

Department of Physics, York University, Ontario, Canada M3J 1P3 ${ }^{a}$ 
${ }^{1}$ also affiliated with University College London, UK

2 also at University of Hamburg, Germany, Alexander von Humboldt Fellow

${ }^{3}$ retired

${ }^{4}$ now at Univ. of Wuppertal, Germany

${ }^{5}$ formerly U. Meyer

${ }^{6}$ supported by Chonnam National University in 2005

7 supported by a scholarship of the World Laboratory Björn Wiik Research Project

${ }^{8}$ supported by the research grant no. 1 P03B 04529 (2005-2008)

${ }^{9}$ now at DESY group FEB, Hamburg, Germany

${ }^{10}$ now at Argonne National Laboratory, Argonne, IL, USA

11 also at Institut of Theoretical and Experimental Physics, Moscow, Russia

12 also at INP, Cracow, Poland

13 on leave of absence from FPACS, AGH-UST, Cracow, Poland

14 partly supported by Moscow State University, Russia

15 also affiliated with DESY

${ }^{16}$ now at ICEPP, University of Tokyo, Japan

17 also at University of Tokyo, Japan

18 Ramón y Cajal Fellow

19 partly supported by Russian Foundation for Basic Research grant no. 05-02-39028NSFC-a

${ }^{20}$ now at CERN, Geneva, Switzerland

${ }^{21}$ PPARC Postdoctoral Research Fellow

22 EU Marie Curie Fellow

23 partially supported by Warsaw University, Poland

24 also at Max Planck Institute, Munich, Germany, Alexander von Humboldt Research Award

${ }^{25}$ now at KEK, Tsukuba, Japan

${ }^{26}$ Department of Radiological Science

27 PPARC Advanced fellow

28 also at Łódź University, Poland

${ }^{29}$ Łódź University, Poland

30 supported by the Polish Ministry for Education and Science grant no. 1 P03B 12629

31 supported by the Polish Ministry for Education and Science grant no. 1 P03B 14129

$\dagger$ deceased 
a supported by the Natural Sciences and Engineering Research Council of Canada (NSERC)

$b$ supported by the German Federal Ministry for Education and Research (BMBF), under contract numbers HZ1GUA 2, HZ1GUB 0, HZ1PDA 5, HZ1VFA 5

c supported in part by the MINERVA Gesellschaft für Forschung GmbH, the Israel Science Foundation (grant no. 293/02-11.2) and the U.S.-Israel Binational Science Foundation

$d \quad$ supported by the German-Israeli Foundation and the Israel Science Foundation

$e \quad$ supported by the Italian National Institute for Nuclear Physics (INFN)

$f$ supported by the Japanese Ministry of Education, Culture, Sports, Science and Technology (MEXT) and its grants for Scientific Research

$g \quad$ supported by the Korean Ministry of Education and Korea Science and Engineering Foundation

$h$ supported by the Netherlands Foundation for Research on Matter (FOM)

$i$ supported by the Polish State Committee for Scientific Research, grant no. 620/E-77/SPB/DESY/P-03/DZ 117/2003-2005 and grant no. 1P03B07427/2004-2006

$j$ partially supported by the German Federal Ministry for Education and Research $(\mathrm{BMBF})$

$k \quad$ supported by RF Presidential grant N 1685.2003.2 for the leading scientific schools and by the Russian Ministry of Education and Science through its grant for Scientific Research on High Energy Physics

$l$ supported by the Spanish Ministry of Education and Science through funds provided by CICYT

$m$ supported by the Particle Physics and Astronomy Research Council, UK

$n$ supported by the US Department of Energy

o supported by the US National Science Foundation

$p \quad$ supported by the Polish Ministry of Scientific Research and Information Technology, grant no. 112/E-356/SPUB/DESY/P-03/DZ 116/2003-2005 and 1 P03B 06527

$q$ supported by FNRS and its associated funds (IISN and FRIA) and by an Inter-University Attraction Poles Programme subsidised by the Belgian Federal Science Policy Office

$r$ supported by the Malaysian Ministry of Science, Technology and Innovation/Akademi Sains Malaysia grant SAGA 66-02-03-0048 


\section{Introduction}

A well established testing ground for perturbative QCD (pQCD) is jet production in neutral current (NC) deep inelastic ep scattering (DIS) at high $Q^{2}$, where $Q^{2}$ is the negative of the square of the virtuality of the exchanged boson $V^{*}(V=\gamma, Z)$. The parton-model process $\left(V^{*} q \rightarrow q\right)$ in DIS gives rise to final states containing one jet of high transverse energy, the so-called 'current jet'. At leading order (LO) in $\alpha_{s}$, the bosongluon-fusion $\left(V^{*} g \rightarrow q \bar{q}\right)$ and QCD-Compton-scattering $\left(V^{*} q \rightarrow q g\right)$ processes give rise to two hard jets.

The hadronic final state in NC DIS may consist of jets with high transverse energy, $E_{T}^{\text {jet }}$, produced in the hard-scattering process, accompanied by the remnant (beam jet) of the incoming proton. For the analysis of these types of process, the Breit frame [1] is advantageous, since it provides a maximal separation between the products of the beam fragmentation and the hard jets. In this frame, the exchanged virtual boson is purely space-like, with 3 -momentum $\boldsymbol{q}=(0,0,-Q)$, and is collinear with the incoming parton. In the parton-model process, the virtual boson $V^{*}$ is absorbed by the struck quark, which is back-scattered with zero transverse momentum with respect to the $V^{*}$ direction, whereas the beam jet follows the direction of the initial struck quark. QCD-Compton and boson-gluon-fusion events, on the other hand, are characterised by two partons in the final state with possibly non-vanishing transverse momentum with respect to the $V^{*}$ direction. Thus, while retaining hard QCD processes at leading order in $\alpha_{s}$, the contribution from the parton-model process can be suppressed by requiring the production of jets with high transverse energy in the Breit frame. Therefore, measurements of inclusivejet events at high $E_{T}^{\text {jet }}$ or dijet events in the Breit frame are directly sensitive to hard QCD processes, allowing direct tests of the pQCD predictions and of the proton parton distribution functions (PDFs).

Jet cross sections in NC DIS have been measured previously at HERA. Inclusive-jet [2-4], dijet [5] and multijet [6] production have been used to extract values of $\alpha_{s}$. A previous dijet analysis [7] has been used to test the gluon density extracted from global fits. Results from inclusive-jet and dijet measurements [3] have been used to constrain the gluon density in the proton. Recently, jet cross sections in NC DIS [2] and photoproduction [8] have been included in a new NLO QCD analysis to extract the proton PDFs [9], resulting in a significant reduction of the uncertainty on the gluon density at medium and high momentum fractions.

This paper presents new measurements of differential dijet cross sections as functions of $Q^{2}$, of the mean jet transverse energy of the dijet system in the Breit frame, $\bar{E}_{T}$, of the dijet invariant mass, $M_{\mathrm{jj}}$, of the half-difference of the jet pseudorapidities in the Breit frame, $\eta^{\prime}=\left|\eta_{\mathrm{B}}^{\mathrm{jet} 1}-\eta_{\mathrm{B}}^{\mathrm{jet} 2}\right| / 2$, and of the fraction of the proton momentum taken by the 
interacting parton, $\xi=x_{\mathrm{Bj}}\left(1+M_{\mathrm{jj}}^{2} / Q^{2}\right)$. Here, $x_{\mathrm{Bj}}$ is the Bjorken scaling variable that defines, for the parton-model process, the fraction of proton momentum carried by the struck parton. Measurements of the dijet cross section as a function of $\xi$ are also shown for different regions of $Q^{2}$. Also presented are measurements of the inclusive-jet cross section as a function of the jet transverse energy in the Breit frame, $E_{T, \mathrm{~B}}^{\mathrm{jet}}$, in different regions of $Q^{2}$. The analyses are based on data samples with more than twice the statistics of the previous studies $[2,7]$. These measurements probe an extended kinematic regime with respect to previous analyses due to the increase in the proton beam energy. The improvement in the experimental uncertainties obtained here will facilitate a more precise determination of the gluon density in the proton at high $\xi$.

\section{Experimental set-up}

A detailed description of the ZEUS detector can be found elsewhere [10,11]. A brief outline of the components that are most relevant for this analysis is given below.

Charged particles are tracked in the central tracking detector (CTD) [12], which operates in a magnetic field of $1.43 \mathrm{~T}$ provided by a thin superconducting solenoid. The CTD consists of 72 cylindrical drift-chamber layers, organized in nine superlayers covering the polar-angle ${ }^{1}$ region $15^{\circ}<\theta<164^{\circ}$. The transverse-momentum resolution for full-length tracks can be parameterised as $\sigma\left(p_{T}\right) / p_{T}=0.0058 p_{T} \oplus 0.0065 \oplus 0.0014 / p_{T}$, with $p_{T}$ in $\mathrm{GeV}$. The tracking system was used to measure the interaction vertex with a typical resolution along (transverse to) the beam direction of $0.4(0.1) \mathrm{cm}$ and to cross-check the energy scale of the calorimeter.

The high-resolution uranium-scintillator calorimeter (CAL) [13] covers $99.7 \%$ of the total solid angle and consists of three parts: the forward (FCAL), the barrel (BCAL) and the rear (RCAL) calorimeters. Each part is segmented into towers and subdivided in depth into one electromagnetic section and either one (in RCAL) or two (in FCAL and BCAL) hadronic sections. The smallest subdivision of the calorimeter is called a cell. Under test-beam conditions, the CAL single-particle relative energy resolutions were $\sigma(E) / E=$ $0.18 / \sqrt{E}$ for electrons and $\sigma(E) / E=0.35 / \sqrt{E}$ for hadrons, with $E$ in $\mathrm{GeV}$.

The luminosity was measured from the rate of the bremsstrahlung process $e p \rightarrow e \gamma p$. The resulting small-angle energetic photons were measured by the luminosity monitor [14], a

\footnotetext{
${ }^{1}$ The ZEUS coordinate system is a right-handed Cartesian system, with the $Z$ axis pointing in the proton beam direction, referred to as the "forward direction", and the $X$ axis pointing left towards the centre of HERA. The coordinate origin is at the nominal interaction point. The polar angle, $\theta$, and the azimuthal angle, $\varphi$, are defined with respect to the proton beam direction. The pseudorapidity is defined as $\eta=-\ln \left(\tan \frac{\theta}{2}\right)$.
} 
lead-scintillator calorimeter placed in the HERA tunnel at $Z=-107 \mathrm{~m}$.

\section{Data selection and jet search}

The data used in this analysis were collected during the period 1998-2000, when HERA operated with protons of energy $E_{p}=920 \mathrm{GeV}$ and electrons or positrons ${ }^{2}$ of energy $E_{e}=27.5 \mathrm{GeV}$, and correspond to an integrated luminosity of $81.7 \pm 1.8 \mathrm{pb}^{-1}$.

A three-level trigger system was used to select events online $[11,15]$. At the third level, NC DIS events were accepted on the basis of the identification of a scattered-electron candidate using localised energy depositions in the CAL. An independent trigger selection which required at least one (two) jet(s) with transverse energies above 10(6) GeV and pseudorapidities below 2.5 was used to measure the fraction of scattered electrons that gave a trigger signal. The efficiency of the trigger selection based on the scattered-electron candidate was found to be above $99 \%$.

Events were selected offline using criteria similar to those reported previously [2]. The main steps are briefly listed below. The inclusive-jet measurement is based on CAL cells for the reconstruction of the kinematic and jet variables, whereas the dijet analysis uses a combination of track and CAL information [16]. The selected combinations of tracks and CAL clusters are referred to as Energy Flow Objects (EFOs).

The scattered-electron candidate was identified from the pattern of energy deposits in the CAL [17]. The energy $\left(E_{e}^{\prime}\right)$ and polar angle $\left(\theta_{e}\right)$ of the electron candidate were determined from the CAL measurements. The kinematic variables $Q^{2}$ and $x_{\mathrm{Bj}}$ were reconstructed using the double angle (DA) method [18]. This method uses $\theta_{e}$ and the angle $\gamma_{h}$, which is equivalent to the angle of the scattered quark in the quark-parton model. The angle $\gamma_{h}$, defined as

$$
\cos \gamma_{h}=\frac{(1-y) x_{\mathrm{Bj}} E_{p}-y E_{e}}{(1-y) x_{\mathrm{Bj}} E_{p}+y E_{e}}
$$

was reconstructed using the hadronic final state [18], where $y=Q^{2} / x_{\mathrm{Bj}} s$ and $s$ is the centre-of-mass energy.

The following requirements were imposed on the data sample for both the inclusive-jet and dijet analyses:

- an electron candidate of energy $E_{e}^{\prime}>10 \mathrm{GeV}$. This requirement ensured a high and well understood electron-finding efficiency and suppressed background from photoproduction events, in which the scattered electron escaped down the rear beampipe;

\footnotetext{
${ }^{2}$ Here and in the following, the term "electron" denotes generically both the electron and the positron.
} 
- the total energy not associated with the electron candidate within a cone of radius 0.7 units in the pseudorapidity-azimuth $(\eta-\varphi)$ plane around the electron direction should be less than $10 \%$ of the electron energy. This condition removed photoproduction and DIS events in which part of a jet was falsely identified as the scattered electron;

- the vertex position along the beam axis should be in the range $|Z|<34 \mathrm{~cm}$. This condition helped to select events consistent with ep interactions;

- $P_{T, \text { miss }} / \sqrt{E_{T}}<2.5 \mathrm{GeV}^{1 / 2}$, where $P_{T, \text { miss }}$ is the missing transverse momentum as measured with the CAL and $E_{T}$ is the total transverse energy in the CAL. This cut removed cosmic-ray events and beam-related background;

- $Q^{2}>125 \mathrm{GeV}^{2}$;

- $-0.65<\cos \gamma_{h}<0.65$. The lower limit avoided a region with limited acceptance due to the requirement on the energy of the scattered electron, while the upper limit was chosen to ensure good reconstruction of the jets in the Breit frame.

In addition, the following selection requirements were imposed for the inclusive-jet analysis:

- $y_{e}<0.95$, where $y_{e}=1-E_{e}^{\prime}\left(1-\cos \theta_{e}\right) /\left(2 E_{e}\right)$. This condition removed events in which fake electron candidates were found in the FCAL;

- if the polar angle of the scattered electron was in the range $30^{\circ}<\theta_{e}<140^{\circ}$, it was required that the fraction of the electron energy within a cone of radius 0.3 units in the $\eta-\varphi$ plane around the electron direction should be larger than 0.9 ; for $\theta_{e}<30^{\circ}$, the cut was raised to 0.98 . This condition removed events in which jets were misidentified as electrons;

- the event was not consistent with elastic Compton scattering $(e p \rightarrow e \gamma p)$, namely no second electromagnetic energy cluster above $10 \mathrm{GeV}$ was allowed when the rest of the CAL energy, besides the two electromagnetic energy clusters, was below $4 \mathrm{GeV}$;

- $38<\left(E-P_{Z}\right)<65 \mathrm{GeV}$, where $E$ is the total energy, $E=\sum_{i} E_{i}$, and $P_{Z}$ is the $Z$-component of the vector $\boldsymbol{P}=\sum_{i} \boldsymbol{p}_{\boldsymbol{i}}$. The sums run over all final-state objects. This cut removed events with large initial-state radiation and further reduced the background from photoproduction.

For the dijet analysis, the following conditions had to be fulfilled:

- $Q^{2}<5000 \mathrm{GeV}^{2}$;

- $45<\left(E-P_{Z}\right)<62 \mathrm{GeV}$.

The $k_{T}$ cluster algorithm [19] was used in the longitudinally invariant inclusive mode [20] to reconstruct jets in the hadronic final state both in data and in Monte Carlo (MC) 
simulated events (see Section 4) assuming massless objects. In data, the algorithm was applied to the final-state objects after excluding the scattered-electron candidate. The jet search was performed in the $\eta-\varphi$ plane of the Breit frame. The jet variables were defined according to the Snowmass convention [21].

After reconstructing the jet variables in the Breit frame, the massless four-momenta were boosted into the laboratory frame, where the transverse energy $\left(E_{T, \mathrm{LAB}}^{\mathrm{jet}}\right)$ and the pseudorapidity $\left(\eta_{\mathrm{LAB}}^{\mathrm{jet}}\right)$ of each jet were calculated. Energy corrections $[2,8,22,23]$ were then applied to the jets in the laboratory frame and propagated into $E_{T, \mathrm{~B}}^{\mathrm{jet}}$ in order to compensate for energy losses in the inactive material in front of the CAL. The following cuts were applied:

- events were removed from the sample if the distance $\Delta$ of any of the jets to the electron candidate in the $\eta-\varphi$ plane of the laboratory frame was smaller than 1 unit, $\Delta=\sqrt{\left(\eta_{\mathrm{LAB}}^{\text {jet }}-\eta^{e}\right)^{2}+\left(\varphi_{\mathrm{LAB}}^{\mathrm{jet}}-\varphi^{e}\right)^{2}}<1$, where $\varphi^{e}$ and $\eta^{e}$ are the azimuthal angle and pseudorapidity of the scattered electron, respectively. This requirement removed some background from photoproduction and improved the purity of the sample;

- events were removed from the sample if a jet was in the backward region of the detector $\left(\eta_{\mathrm{IAB}}^{\text {jet }}<-2\right)$. This requirement removed events in which a radiated photon from the electron was misidentified as a jet in the Breit frame;

- $-2<\eta_{\mathrm{B}}^{\text {jet }}<1.5$. This cut restricted the jets to a region with large acceptance.

The inclusive-jet sample was then selected using the following conditions:

- $E_{T, \mathrm{LAB}}^{\mathrm{jet}}>2.5 \mathrm{GeV}$. This cut removed a small number of jets for which the uncertainty on the energy correction was large;

- $E_{T, \mathrm{~B}}^{\mathrm{jet}}>8 \mathrm{GeV}$.

For the dijet analysis, the following further requirements were imposed:

- $E_{T, \mathrm{LAB}}^{\mathrm{jet}}>3 \mathrm{GeV}$;

- $\eta_{\mathrm{I} A \mathrm{~B}}^{\mathrm{jet}}<2.5$. This condition restricted the jets to the region in which the reconstruction of the jet variables was optimal;

- the two highest- $E_{T, \mathrm{~B}}^{\mathrm{jet}}$ jets in an event, ordered according to $E_{T, \mathrm{~B}}^{\mathrm{jet}}$, were required to satisfy $E_{T, \mathrm{~B}}^{\mathrm{jet} 1}>12 \mathrm{GeV}$ and $E_{T, \mathrm{~B}}^{\mathrm{jet} 2}>8 \mathrm{GeV}$.

The selected sample of inclusive-jet (dijet) events consisted of 19908 (3868) events. 


\section{Monte Carlo simulation and acceptance corrections}

Samples of MC events were generated to determine the response of the detector to jets of hadrons and the correction factors necessary to obtain the hadron-level jet cross sections. The hadron level is defined in terms of hadrons with lifetime $\tau \geq 10 \mathrm{ps}$. The generated events were passed through the GEANT 3.13-based [24] ZEUS detector- and trigger-simulation programs [11]. They were reconstructed and analysed by the same program chain as the data.

Neutral current DIS events including radiative effects were simulated using the HERACLES 4.6.1 [25] program with the DJANGOH 1.1 [26] interface to the hadronisation programs. HERACLES includes corrections for initial- and final-state radiation, vertex and propagator terms, and two-boson exchange. The QCD cascade is simulated using the colour-dipole model (CDM) [27] including the LO QCD diagrams as implemented in ARIADNE 4.08 [28] and, alternatively, with the MEPS model of LEPTO 6.5 [29]. The CTEQ5D [30] proton PDFs were used for these simulations. Fragmentation into hadrons is performed using the Lund string model [31] as implemented in JETSET 7.4 [32].

The jet search was performed on the MC events using the energy measured in the CAL cells or EFOs in the same way as for the data. The same jet algorithm was also applied to the final-state particles (hadron level) and to the partons available after the parton shower (parton level).

The data were corrected to the hadron level and for QED-radiative effects using bin-bybin correction factors obtained from the MC samples. For this approach to be valid, the uncorrected distributions of the data must be well described by the MC simulations. This condition was in general satisfied by both the Ariadne and Lepto MC. The Ariadne model gave a slightly better description of the inclusive-jet data and was thus used as the

default model; LEPTO was then used to estimate the systematic effect on the correction procedure due to the parton-shower model (see Section 6). In contrast to this, the dijet distributions, shown in Fig. 1, were slightly better described by the LEPTO model; here, ARIADNE was therefore used for systematic checks. In all cases, the correction factors differed from unity by typically 10\%. These correction factors took into account the efficiency of the trigger, the selection criteria and the purity and efficiency of the jet reconstruction.

\section{$5 \quad$ NLO QCD calculations}

The measurements were compared with NLO QCD $\left(\mathcal{O}\left(\alpha_{s}^{2}\right)\right)$ calculations obtained using the program DisEnT [33]. The calculations were performed in the $\overline{\mathrm{MS}}$ renormalisation and 
factorisation schemes using a generalised version [33] of the subtraction method [34]. The number of flavours was set to five and the factorisation scale was chosen to be $\mu_{F}=Q$. Calculations with different choices of the renormalisation scale, $\mu_{R}$, were performed: the default choice was $\mu_{R}^{2}=\left(E_{T, \mathrm{~B}}^{\mathrm{jet}}\right)^{2}$ for the inclusive-jet analysis; cross checks were performed using $\mu_{R}^{2}=Q^{2}$ and $\mu_{R}^{2}=Q^{2}+\bar{E}_{T, \mathrm{~B}}^{2}$, where $\bar{E}_{T, \mathrm{~B}}^{2}$ is the mean transverse energy of the selected jets in an event. For the dijet analysis $\mu_{R}^{2}=Q^{2}+\bar{E}_{T}^{2}$ was used as default, since the resulting predictions describe the data better than either scale alone; here the effects of using either $Q^{2}$ or $\bar{E}_{T}^{2}$ were investigated. The effects on the NLO calculations of using different choices for $\mu_{R}$ were found to be smaller than the uncertainties described below. The strong coupling constant was calculated at two loops with $\Lambda \frac{(5)}{\mathrm{MS}}=226 \mathrm{MeV}$, corresponding to $\alpha_{s}\left(M_{Z}\right)=0.118$. The calculations were performed using the CTEQ6 [35] parameterisations of the proton PDFs. The $k_{T}$ cluster algorithm was also applied to the partons in the events generated by DISENT in order to obtain the jet cross-section predictions.

Since the measurements refer to jets of hadrons, whereas the NLO QCD calculations refer to jets of partons, the predictions were corrected to the hadron level using the MC models. The multiplicative correction factor $\left(C_{\text {had }}\right)$ was defined as the ratio of the cross section for jets of hadrons over that for jets of partons, estimated by using the MC programs described in Section 4. The ratios obtained with ARIADNE and LEPTO were averaged to obtain the value of $C_{\text {had }}$. This value differs from unity by approximately $5(5-10) \%$ for the inclusive-jet (dijet) analysis.

The NLO QCD predictions for the inclusive-jet analysis were also corrected for the $Z^{0}$ exchange contribution by using MC simulated events with and without $Z^{0}$ exchange. This correction is not required for the dijet analysis owing to the restricted $Q^{2}$ range.

Several sources of uncertainty in the theoretical predictions were considered:

- the uncertainty on the NLO QCD calculations due to terms beyond NLO, estimated by varying $\mu_{R}$ by a factor of two up and down, was below $\pm 7 \%$ at low $Q^{2}$ and low $E_{T, \mathrm{~B}}^{\mathrm{jet}}$ and decreased to below $\pm 5 \%$ in the highest- $Q^{2}$ region for the inclusive-jet analysis. For the dijet measurement, the average uncertainty was about $\pm 10 \%$, with the largest values approaching $\pm 20 \%$ at low $\xi$ and low $Q^{2}$ values.

- the uncertainty on the NLO QCD calculations due to that on $\alpha_{s}\left(M_{Z}\right)$ was estimated by repeating the calculations using two additional sets of proton PDFs, CTEQ6A114 and CTEQ6A122 [36], determined assuming $\alpha_{s}\left(M_{Z}\right)=0.114$ and 0.122 , respectively. The difference between the calculations using these sets and CTEQ6 was scaled by a factor of 0.68 to reflect the uncertainty on $\alpha_{s}$ [37]. The resulting uncertainty in the cross sections was typically below $\pm 4 \%$;

- the uncertainty on the modelling of the parton shower was estimated as half the 
difference between the multiplicative correction factors calculated from the LEPTO and ARIADne models. The resulting uncertainty on the cross sections was typically less than $3 \%$;

- the uncertainty on the NLO calculations due to the proton PDFs was estimated by repeating the calculations using 40 additional sets from the CTEQ6 analysis, which takes into account the statistical and correlated systematic experimental uncertainties of each data set used in the determination of the proton PDFs. The resulting uncertainty in the cross sections was about $\pm 4 \%$ at low $Q^{2}$ and decreased to around $\pm 2 \%$ at high $Q^{2}$;

- the uncertainty of the calculations in the value of $\mu_{F}$ was estimated by repeating the calculations with $\mu_{F}=Q / 2$ and $2 Q$. The variation of the calculations was negligible.

The total theoretical uncertainty was obtained by adding in quadrature the individual uncertainties listed above. Figures 2 and 3 show an overview of various theoretical uncertainties for both the inclusive-jet and the dijet analysis.

The gluon-induced contribution to the cross section as a function of $E_{T, \mathrm{~B}}^{\mathrm{jet}}$ for the inclusivejet analysis and of $\log _{10} \xi$ for the dijet analysis in various regions of $Q^{2}$ is shown in Figs. 4 and 5. The gluon fraction varies between $70 \%$ at low $Q^{2}$ for both low $\xi$ and low $E_{T, \mathrm{~B}}^{\text {jet }}$ and less than $10 \%$ for the lowest $E_{T, \mathrm{~B}}^{\mathrm{jet}}$ at high values of $Q^{2}$. For the dijet analysis, the gluon contribution is always at least 30\%. In addition, Fig. 6 shows, separately for the inclusive-jet and dijet analyses, the gluon-induced contribution to the cross section as a function of $Q^{2}$ together with the uncertainties as taken from the CTEQ6 analysis.

\section{$6 \quad$ Experimental uncertainties}

The following sources of systematic uncertainty were considered for the measured cross sections:

- the uncertainty in the absolute energy scale of the jets was estimated to be $\pm 1 \%$ for $E_{T, \mathrm{LAB}}^{\mathrm{jet}}>10 \mathrm{GeV}$ and $\pm 3 \%$ for lower $E_{T, \mathrm{LAB}}^{\mathrm{jet}}$ values $[8,22,38]$. The resulting uncertainty on the cross sections was about $\pm 5 \%$ and increased to approximately $\pm 10 \%$ in certain regions of the dijet phase space;

- the uncertainty in the absolute energy scale of the electron candidate was estimated to be $\pm 1 \%$ [39]. The resulting uncertainty was below $\pm 1 \%$;

- the differences in the results obtained by using either ARIADNE or LEPTO to correct the data for detector effects were typically below $\pm 8 \%$;

- the analysis was repeated using an alternative technique [40] to select the scatteredelectron candidate. The resulting uncertainty was typically below $\pm 3 \%$; 
- the $E_{T, \mathrm{LAB}}^{\mathrm{jet}}$ cut was raised to $4(4.5) \mathrm{GeV}$ for the inclusive-jet (dijet) analysis. The resulting uncertainty was typically smaller than $\pm 1 \%$;

- the cut in $\eta_{\mathrm{L} \text { AB }}^{\text {jet }}$ used to suppress the contamination due to photons falsely identified as jets in the Breit frame was changed to -3 and to -1.5 . The resulting uncertainty was typically below $\pm 1 \%$;

- the uncertainty due to the selection cuts was estimated by varying the values of the cuts within the resolution of each variable. The effect on the cross sections was typically below $\pm 2 \%$;

- the uncertainty on the cross sections due to that in the simulation of the trigger was negligible.

The systematic uncertainties not associated with the absolute energy scale of the jets were added in quadrature to the statistical uncertainties and are shown in figures 7 to 12 as error bars. The uncertainty due to the absolute energy scale of the jets is shown separately as a shaded band in each of these figures, due to the large bin-to-bin correlation. In addition, there was an overall normalisation uncertainty of $2.2 \%$ from the luminosity determination, which is not included in the figures.

\section{Results}

\subsection{Dijet differential cross sections}

Dijet cross sections were measured in the kinematic region $125<Q^{2}<5000 \mathrm{GeV}^{2}$ and $\left|\cos \gamma_{h}\right|<0.65$. These cross sections correspond to the two highest- $E_{T, \mathrm{~B}}^{\mathrm{jet}}$ jets in each event with $E_{T, \mathrm{~B}}^{\text {jet1 }}>12 \mathrm{GeV}, E_{T, \mathrm{~B}}^{\mathrm{jet} 2}>8 \mathrm{GeV}$ and $-2<\eta_{\mathrm{B}}^{\text {jet }}<1.5$ and were corrected for detector and QED radiative effects as described in Section 4.

The measured cross sections as functions of $Q^{2}, x_{\mathrm{Bj}}, \bar{E}_{T}, M_{\mathrm{j} . \mathrm{j}}, \eta^{\prime}$ and $\log _{10} \xi$ are shown in Figs. 7 and 8 and are listed in Tables 1 to 6 . In these and subsequent figures, each data point is plotted at the abscissa at which the differential cross section was equal to its bin-averaged value, according to the NLO QCD calculation. The data distribution as a function of $Q^{2}$ exhibits a fall-off of more than two orders of magnitude within the range studied. The $Q^{2}$-restricted range implicitly limits the $\xi$ range of the measurements to $0.009 \lesssim \xi \lesssim 0.37$, a region of phase space where these measurements can significantly constrain the gluon density in the proton. The dijet cross sections as functions of $\bar{E}_{T}$ and $M_{\mathrm{jj}}$ are particularly suited to test the matrix elements in the perturbative calculations. The measured distributions show a steep fall-off of more than two orders of magnitude within the measured range. 
The NLO QCD calculations with $\mu_{R}^{2}=Q^{2}+\bar{E}_{T}^{2}$ are compared to the data in Figs. 7 and 8. They give a good description of the data. The calculations with $\mu_{R}^{2}=Q^{2}$ or $\mu_{R}^{2}=\bar{E}_{T}^{2}$ are included in Figs. 7 and 8 as well. At low $Q^{2}$, low $M_{\mathrm{jj}}$ and low $\bar{E}_{T}$, the theoretical uncertainty is dominated by the effect of the variation of the renormalisation scale. In the case of the dijet cross sections as functions of $\bar{E}_{T}$ and $M_{\mathrm{jj}}$, the theoretical uncertainty is smaller than the experimental at high values of $\bar{E}_{T}$ and $M_{\mathrm{jj} .}$. The excellent agreement between data and theory for these distributions demonstrates the validity of the description of the dynamics of dijet production by pQCD at $\mathcal{O}\left(\alpha \alpha_{s}^{2}\right)$.

\subsection{Dijet and inclusive-jet differential cross sections in different regions of $Q^{2}$}

Figure 9 shows the measured dijet cross section as a function of $\log _{10} \xi$ in different regions of $Q^{2}$. The cross sections are also given in Table 7 . The requirement that two jets with high transverse energy be observed in the final state suppresses the cross section in the low- $\xi$ region, so that the measured cross section rises as $\log _{10} \xi$ increases. At high values of $\log _{10} \xi$, the decrease of the cross section reflects the decrease of the gluon and quark densities at high $\xi$.

The NLO QCD predictions with $\mu_{R}^{2}=Q^{2}+\bar{E}_{T}^{2}$ are compared to the measurements in Fig. 10 using the relative difference of the measured differential cross sections to the NLO calculations; the uncertainty of the calculation is also shown in the figure. The predictions of NLO QCD give a good description of the data. Also indicated are the predictions using $\mu_{R}^{2}=Q^{2}$ or $\mu_{R}^{2}=\bar{E}_{T}^{2}$.

Inclusive-jet cross sections were measured in the kinematic region $Q^{2}>125 \mathrm{GeV}^{2}$ and $\left|\cos \gamma_{h}\right|<0.65$. These cross sections include every jet of hadrons in the event with $E_{T, \mathrm{~B}}^{\text {jet }}>8 \mathrm{GeV}$ and $-2<\eta_{\mathrm{B}}^{\text {jet }}<1.5$ and were corrected for detector and QED radiative effects. The cross sections for different regions of $Q^{2}$ as a function of $E_{T, \mathrm{~B}}^{\mathrm{jet}}$ are presented in Fig. 11 and Table 8. The measured cross sections exhibit a steep fall-off within the $E_{T, \mathrm{~B}}^{\text {jet }}$ range considered. As $Q^{2}$ increases, the $E_{T, \mathrm{~B}}^{\mathrm{jet}}$ dependence of the cross section becomes less steep.

The NLO QCD predictions with $\mu_{R}^{2}=\left(E_{T, \mathrm{~B}}^{\mathrm{jet}}\right)^{2}$ are compared to the measurements in Fig. 11. They give a good description of the data. To study the scale dependence, calculations using $\mu_{R}^{2}=Q^{2}$ or $\mu_{R}^{2}=Q^{2}+\bar{E}_{T, \mathrm{~B}}^{2}$ are also compared to the data in Fig. 11; they provide an approximately equally good description of the data.

Figure 12 shows the relative difference of the measured differential cross sections to the NLO QCD calculations with $\mu_{R}^{2}=\left(E_{T, \mathrm{~B}}^{\mathrm{jet}}\right)^{2}$. The uncertainty of the NLO QCD calculations 
and the results of the theory calculations using either $\mu_{R}^{2}=Q^{2}$ or $\mu_{R}^{2}=Q^{2}+\bar{E}_{T, \mathrm{~B}}^{2}$ are also shown. The data are well described by the predictions.

As indicated in Section 5, the theoretical uncertainties in both the inclusive-jet and dijet regimes are dominated by the contribution from higher orders as estimated by varying the renormalisation scale. As was shown in Figs. 2 and 3, the scale uncertainty decreases as $Q^{2}$ increases; it is also lower for high values of $\xi$ than for low values of this variable. The contribution from the PDF uncertainty is approximately constant in all variables and non-negligible. Especially at high values of $\xi\left(E_{T, \mathrm{~B}}^{\text {jet }}\right)$ for the dijet (inclusive-jet) analysis, the PDF uncertainty is even dominant. In these regions, in which the gluon-induced contribution is still substantial (see Figs. 4 to 6), the data will be able to constrain further the gluon density in the proton.

\section{Summary and conclusions}

Inclusive-jet and dijet cross sections have been measured at high $Q^{2}$ values at HERA for $\sqrt{s}=318 \mathrm{GeV}$. The jets were reconstructed using the $k_{T}$ cluster algorithm in the longitudinally invariant inclusive mode in the Breit frame. The dijet cross sections were measured as functions of $Q^{2}, x_{\mathrm{Bj}}, \bar{E}_{T}, M_{\mathrm{jij}}, \eta^{\prime}$ and $\xi$. In addition, inclusive-jet and dijet measurements were performed in different regions of $Q^{2}$ as functions of $E_{T, \mathrm{~B}}^{\mathrm{jet}}$ and $\xi$, respectively. The data are well described by the NLO QCD predictions. The cross sections in different regions of $Q^{2}$ are shown to be sensitive to the gluon density of the proton. The precise measurements obtained here are therefore of particular relevance for improving the determination of the gluon density in future $\mathrm{QCD}$ fits.

\section{Acknowledgements}

We thank the DESY Directorate for their strong support and encouragement. The remarkable achievements of the HERA machine group were essential for the successful completion of this work and are greatly appreciated. We are grateful for the support of the DESY computing and network services. The design, construction and installation of the ZEUS detector have been made possible owing to the ingenuity and effort of many people from DESY and home institutes who are not listed as authors. 


\section{References}

[1] R.P. Feynman, Photon-Hadron Interactions. Benjamin, New York, (1972);

K.H. Streng, T.F. Walsh and P.M. Zerwas, Z. Phys. C 2, 237 (1979).

[2] ZEUS Coll., S. Chekanov et al., Phys. Lett. B 547, 164 (2002).

[3] H1 Coll., C. Adloff et al., Eur. Phys. J. C 19, 289 (2001).

[4] H1 Coll., C. Adloff et al., Phys. Lett. B 542, 193 (2002).

[5] ZEUS Coll., J. Breitweg et al., Phys. Lett. B 507, 70 (2001).

[6] ZEUS Coll., S. Chekanov et al., Eur. Phys. J. C 44, 183 (2005).

[7] ZEUS Coll., S. Chekanov et al., Eur. Phys. J. C 23, 13 (2002).

[8] ZEUS Coll., S. Chekanov et al., Eur. Phys. J. C 23, 615 (2002).

[9] ZEUS Coll., S. Chekanov et al., Eur. Phys. J. C 42, 1 (2005).

[10] ZEUS Coll., M. Derrick et al., Phys. Lett. B 293, 465 (1992).

[11] ZEUS Coll., U. Holm (ed.), The ZEUS Detector. Status Report (unpublished), DESY (1993), available on http://www-zeus.desy.de/bluebook/bluebook.html.

[12] N. Harnew et al., Nucl. Inst. Meth. A 279, 290 (1989);

B. Foster et al., Nucl. Phys. Proc. Suppl. B 32, 181 (1993);

B. Foster et al., Nucl. Inst. Meth. A 338, 254 (1994).

[13] M. Derrick et al., Nucl. Inst. Meth. A 309, 77 (1991);

A. Andresen et al., Nucl. Inst. Meth. A 309, 101 (1991);

A. Caldwell et al., Nucl. Inst. Meth. A 321, 356 (1992);

A. Bernstein et al., Nucl. Inst. Meth. A 336, 23 (1993).

[14] J. Andruszków et al., Preprint DESY-92-066, DESY, 1992;

ZEUS Coll., M. Derrick et al., Z. Phys. C 63, 391 (1994);

J. Andruszków et al., Acta Phys. Pol. B 32, 2025 (2001).

[15] W.H. Smith, K. Tokushuku and L.W. Wiggers, Proc. Computing in High-Energy Physics (CHEP), Annecy, France, Sept. 1992, C. Verkerk and W. Wojcik (eds.), p. 222. CERN, Geneva, Switzerland (1992). Also in preprint DESY 92-150B.

[16] G.M. Briskin, Diffractive Dissociation in ep Deep Inelastic Scattering. Ph.D. Thesis, Tel Aviv University, DESY-THESIS-1998-036, 1998.

[17] H. Abramowicz, A. Caldwell and R. Sinkus, Nucl. Inst. Meth. A 365, 508 (1995);

R. Sinkus and T. Voss, Nucl. Inst. Meth. A 391, 360 (1997).

[18] S. Bentvelsen, J. Engelen and P. Kooijman, Proc. of the Workshop on Physics at HERA, W. Buchmüller and G. Ingelman (eds.), Vol. 1, p. 23. Hamburg, Germany, 
DESY (1992);

K.C. Höger, ibid., p. 43.

[19] S. Catani et al., Nucl. Phys. B 406, 187 (1993).

[20] S.D. Ellis and D.E. Soper, Phys. Rev. D 48, 3160 (1993).

[21] J.E. Huth et al., Research Directions for the Decade. Proc. of Summer Study on High Energy Physics, 1990, E.L. Berger (ed.), p. 134. World Scientific (1992). Also in preprint FERMILAB-CONF-90-249-E.

[22] ZEUS Coll., S. Chekanov et al., Phys. Lett. B 531, 9 (2002).

[23] ZEUS Coll., S. Chekanov et al., Phys. Lett. B 558, 41 (2003).

[24] R. Brun et al., GEAnt3, Technical Report CERN-DD/EE/84-1, CERN, 1987.

[25] A. Kwiatkowski, H. Spiesberger and H.-J. Möhring, Comp. Phys. Comm. 69, 155 (1992);

H. Spiesberger, An Event Generator for ep Interactions at HERA Including Radiative Processes (Version 4.6), 1996, available on http://www.desy.de/ ${ }^{\sim}$ hspiesb/heracles.html.

[26] K. Charcuła, G.A. Schuler and H. Spiesberger, Comp. Phys. Comm. 81, 381 (1994); H. Spiesberger, HERACles and DJANGOH: Event Generation for ep Interactions at HERA Including Radiative Processes, 1998, available on http://www.desy.de/ ^hspiesb/djangoh.html.

[27] Y. Azimov et al., Phys. Lett. B 165, 147 (1985);

G. Gustafson, Phys. Lett. B 175, 453 (1986);

G. Gustafson and U. Pettersson, Nucl. Phys. B 306, 746 (1988);

B. Andersson et al., Z. Phys. C 43, 625 (1989).

[28] L. Lönnblad, Comp. Phys. Comm. 71, 15 (1992);

L. Lönnblad, Z. Phys. C 65, 285 (1995).

[29] G. Ingelman, A. Edin and J. Rathsman, Comp. Phys. Comm. 101, 108 (1997).

[30] H.L. Lai et al., Eur. Phys. J. C 12, 375 (2000).

[31] B. Andersson et al., Phys. Rep. 97, 31 (1983).

[32] T. Sjöstrand, Comp. Phys. Comm. 82, 74 (1994);

T. Sjöstrand, Comp. Phys. Comm. 39, 347 (1986);

T. Sjöstrand and M. Bengtsson, Comp. Phys. Comm. 43, 367 (1987).

[33] S. Catani and M.H. Seymour, Nucl. Phys. B 485, 291 (1997). Erratum in Nucl. Phys. B 510 (1998) 503.

[34] R.K. Ellis, D.A. Ross and A.E. Terrano, Nucl. Phys. B 178, 421 (1981). 
[35] J. Pumplin et al., JHEP 0207, 012 (2002);

D. Stump et al., JHEP 0310, 046 (2003).

[36] J. Pumplin et al., JHEP 0602, 032 (2006).

[37] S. Bethke, J. Phys. G 26, R27 (2000). Updated in Preprint hep-ex/0407021, 2004.

[38] M. Wing (on behalf of the ZEUS Coll.), Proc. of the 10th International Conference on Calorimetry in High Energy Physics, R. Zhu (ed.), p. 767. Pasadena, USA (2002). Also in preprint hep-ex/0206036.

[39] ZEUS Coll., S. Chekanov et al., Eur. Phys. J. C 21, 443 (2001).

[40] ZEUS Coll., J. Breitweg et al., Eur. Phys. J. C 11, 427 (1999). 


\begin{tabular}{||c|cccc||c||c||}
\hline $\begin{array}{c}Q^{2} \text { bin } \\
\left(\mathrm{GeV}^{2}\right)\end{array}$ & $\begin{array}{c}d \sigma / d Q^{2} \\
\left(\mathrm{pb} / \mathrm{GeV}^{2}\right)\end{array}$ & $\delta_{\text {stat }}$ & $\delta_{\text {syst }}$ & $\delta_{\mathrm{ES}}$ & $C_{\mathrm{QED}}$ & $C_{\text {had }}$ \\
\hline \hline $125-250$ & 0.1183 & \pm 0.0033 & ${ }_{-0.0040}^{+0.0041}$ & ${ }_{-0.0077}^{+0.0081}$ & 0.96 & 0.85 \\
$250-500$ & 0.0589 & \pm 0.0018 & ${ }_{-0.0023}^{+0.0025}$ & ${ }_{-0.0030}^{+0.0032}$ & 0.94 & 0.91 \\
$500-1000$ & 0.02061 & \pm 0.00074 & ${ }_{-0.00094}^{+0.00094}$ & ${ }_{-0.00083}^{+0.00096}$ & 0.93 & 0.92 \\
$1000-2000$ & 0.00602 & \pm 0.00028 & ${ }_{-0.00018}^{+0.00018}$ & ${ }_{-0.00013}^{+0.00014}$ & 0.91 & 0.95 \\
$2000-5000$ & 0.001189 & \pm 0.000075 & ${ }_{-0.000083}^{+0.00083}$ & ${ }_{-0.000020}^{+0.000025}$ & 0.96 & 0.96
\end{tabular}

Table 1: Dijet cross-section $d \sigma / d Q^{2}$ for jets of hadrons in the Breit frame selected with the longitudinally invariant $k_{T}$ cluster algorithm. The statistical, uncorrelated systematic and jet-energy-scale (ES) uncertainties are shown separately. The multiplicative corrections applied to the data to correct for $Q E D$ radiative effects, $C_{\mathrm{QED}}$, and the corrections for hadronisation effects to be applied to the parton-level NLO $Q C D$ calculations, $C_{\mathrm{had}}$, are shown in the last two columns.

\begin{tabular}{||c|cccc||c||c||}
\hline$x_{\mathrm{Bj}}$ bin & $d \sigma / d x_{\mathrm{Bj}}$ & & & & \\
& $(\mathrm{pb})$ & $\delta_{\text {stat }}$ & $\delta_{\text {syst }}$ & $\delta_{\mathrm{ES}}$ & $C_{\mathrm{QED}}$ & $C_{\mathrm{had}}$ \\
\hline \hline $0.0001-0.01$ & 1599 & \pm 45 & ${ }_{-52}^{+54}$ & ${ }_{-92}^{+101}$ & 0.95 & 0.86 \\
$0.01-0.02$ & 1849 & \pm 48 & ${ }_{-37}^{+37}$ & ${ }_{-95}^{+98}$ & 0.94 & 0.93 \\
$0.02-0.035$ & 647 & \pm 24 & ${ }_{-25}^{+26}$ & ${ }_{-23}^{+26}$ & 0.92 & 0.92 \\
$0.035-0.07$ & 141.7 & \pm 7.5 & ${ }_{-4.0}^{+4.0}$ & ${ }_{-2.6}^{+2.9}$ & 0.95 & 0.91 \\
$0.07-0.1$ & 21.1 & \pm 3.2 & ${ }_{-3.3}^{+3.3}$ & ${ }_{-0.1}^{+0.5}$ & 0.89 & 0.90 \\
\hline
\end{tabular}

Table 2: Dijet cross-section $d \sigma / d x_{\mathrm{Bj}}$ for jets of hadrons in the Breit frame selected with the longitudinally invariant $k_{T}$ cluster algorithm. Other details as in the caption to Table 1. 


\begin{tabular}{||c|cccc||c||c||}
\hline $\begin{array}{c}\bar{E}_{T} \text { bin } \\
(\mathrm{GeV})\end{array}$ & $\begin{array}{c}d \sigma / d \bar{E}_{T} \\
(\mathrm{pb} / \mathrm{GeV})\end{array}$ & $\delta_{\text {stat }}$ & $\delta_{\text {syst }}$ & $\delta_{\mathrm{ES}}$ & $C_{\mathrm{QED}}$ & $C_{\text {had }}$ \\
\hline \hline $10-16$ & 5.71 & \pm 0.11 & ${ }_{-0.12}^{+0.12}$ & ${ }_{-0.26}^{+0.30}$ & 0.94 & 0.90 \\
$16-22$ & 1.935 & \pm 0.064 & ${ }_{-0.084}^{+0.086}$ & ${ }_{-0.091}^{+0.081}$ & 0.94 & 0.90 \\
$22-30$ & 0.361 & \pm 0.023 & ${ }_{-0.023}^{+0.023}$ & ${ }_{-0.016}^{+0.017}$ & 0.94 & 0.91 \\
$30-60$ & 0.0371 & \pm 0.0043 & ${ }_{-0.0076}^{+0.0076}$ & ${ }_{-0.0018}^{+0.0021}$ & 0.97 & 0.91
\end{tabular}

Table 3: Dijet cross-section $d \sigma / d \bar{E}_{T}$ for jets of hadrons in the Breit frame selected with the longitudinally invariant $k_{T}$ cluster algorithm. Other details as in the caption to Table 1.

\begin{tabular}{||c|cccc||c||c||}
\hline $\begin{array}{c}M_{\mathrm{jj}} \text { bin } \\
(\mathrm{GeV})\end{array}$ & $\begin{array}{c}d \sigma / d M_{\mathrm{jj}} \\
(\mathrm{pb} / \mathrm{GeV})\end{array}$ & $\delta_{\text {stat }}$ & $\delta_{\text {syst }}$ & $\delta_{\mathrm{ES}}$ & $C_{\mathrm{QED}}$ & $C_{\text {had }}$ \\
\hline \hline $20-32$ & 2.382 & \pm 0.051 & ${ }_{-0.054}^{+0.055}$ & ${ }_{-0.109}^{+0.123}$ & 0.95 & 0.91 \\
$32-45$ & 1.134 & \pm 0.034 & ${ }_{-0.047}^{+0.047}$ & ${ }_{-0.055}^{+0.049}$ & 0.94 & 0.89 \\
$45-65$ & 0.222 & \pm 0.012 & ${ }_{-0.006}^{+0.006}$ & ${ }_{-0.009}^{+0.009}$ & 0.92 & 0.89 \\
$65-120$ & 0.0180 & \pm 0.0021 & ${ }_{-0.0017}^{+0.0017}$ & ${ }_{-0.0009}^{+0.0013}$ & 0.97 & 0.94
\end{tabular}

Table 4: Dijet cross-section $d \sigma / d M_{\mathrm{ji}}$ for jets of hadrons in the Breit frame selected with the longitudinally invariant $k_{T}$ cluster algorithm. Other details as in the caption to Table 1.

\begin{tabular}{||c|cccc||c||c||}
\hline$\eta^{\prime}$ bin & $d \sigma / d \eta^{\prime}$ & & & & \\
& $(\mathrm{pb})$ & $\delta_{\text {stat }}$ & $\delta_{\text {syst }}$ & $\delta_{\mathrm{ES}}$ & $C_{\mathrm{QED}}$ & $C_{\text {had }}$ \\
\hline \hline $0-0.1$ & 87.9 & \pm 3.4 & ${ }_{-3.9}^{+4.0}$ & ${ }_{-3.7}^{+3.7}$ & 0.95 & 0.91 \\
$0.1-0.25$ & 87.0 & \pm 2.8 & ${ }_{-1.6}^{+1.6}$ & ${ }_{-3.7}^{+3.6}$ & 0.94 & 0.94 \\
$0.25-0.45$ & 68.5 & \pm 2.1 & ${ }_{-1.1}^{+1.3}$ & ${ }_{-3.0}^{+3.5}$ & 0.95 & 0.91 \\
$0.45-0.65$ & 42.7 & \pm 1.6 & ${ }_{-1.0}^{+1.0}$ & ${ }_{-2.2}^{+2.5}$ & 0.94 & 0.87 \\
$0.65-1.60$ & 6.07 & \pm 0.28 & ${ }_{-0.52}^{+0.54}$ & ${ }_{-0.34}^{+0.37}$ & 0.91 & 0.86 \\
\hline
\end{tabular}

Table 5: Dijet cross-section $d \sigma / d \eta^{\prime}$ for jets of hadrons in the Breit frame selected with the longitudinally invariant $k_{T}$ cluster algorithm. Other details as in the caption to Table 1. 


\begin{tabular}{||c|cccc||c||c||}
\hline $\log _{10} \xi$ bin & $\begin{array}{c}d \sigma / d \log _{10} \xi \\
(\mathrm{pb})\end{array}$ & $\delta_{\text {stat }}$ & $\delta_{\text {syst }}$ & $\delta_{\mathrm{ES}}$ & $C_{\mathrm{QED}}$ & $C_{\text {had }}$ \\
\hline \hline$-2--1.5$ & 22.22 & \pm 0.78 & ${ }_{-0.71}^{+0.72}$ & ${ }_{-1.17}^{+1.34}$ & 0.95 & 0.88 \\
$-1.5--1.35$ & 74.4 & \pm 2.6 & ${ }_{-2.8}^{+2.8}$ & ${ }_{-3.4}^{+3.2}$ & 0.94 & 0.92 \\
$-1.35--1.1$ & 73.9 & \pm 1.9 & ${ }_{-2.0}^{+2.2}$ & ${ }_{-3.2}^{+3.4}$ & 0.94 & 0.92 \\
$-1.1--0.85$ & 31.0 & \pm 1.3 & ${ }_{-2.4}^{+2.4}$ & ${ }_{-1.4}^{+1.5}$ & 0.94 & 0.87 \\
$-0.85--0.5$ & 3.93 & \pm 0.36 & ${ }_{-0.48}^{+0.48}$ & ${ }_{-0.21}^{+0.32}$ & 0.93 & 0.79 \\
\hline
\end{tabular}

Table 6: Dijet cross-section $d \sigma / d \log _{10} \xi$ for jets of hadrons in the Breit frame selected with the longitudinally invariant $k_{T}$ cluster algorithm. Other details as in the caption to Table 1. 


\begin{tabular}{|c|c|c|c|c|c|c|}
\hline $\log _{10} \xi$ bin & $\begin{array}{c}d \sigma / d \log _{10} \xi \\
(\mathrm{pb})\end{array}$ & $\delta_{\text {stat }}$ & $\delta_{\text {syst }}$ & $\delta_{\mathrm{ES}}$ & $C_{\mathrm{QED}}$ & $C_{\text {had }}$ \\
\hline \multicolumn{7}{|c|}{$125<Q^{2}<250 \mathrm{GeV}^{2}$} \\
\hline$-2.00--1.50$ & 9.17 & \pm 0.48 & $\begin{array}{l}+0.44 \\
-0.44\end{array}$ & $\begin{array}{l}+0.70 \\
-0.62\end{array}$ & 0.97 & 0.83 \\
\hline$-1.50--1.35$ & 25.0 & \pm 1.4 & ${ }_{-1.8}^{+1.8}$ & ${ }_{-0.7}^{+1.4}$ & 0.95 & 0.89 \\
\hline$-1.35--1.10$ & 19.74 & \pm 0.93 & ${ }_{-0.41}^{+0.43}$ & $\begin{array}{l}+1.17 \\
-1.65\end{array}$ & 0.95 & 0.86 \\
\hline$-1.10--0.50$ & 2.52 & \pm 0.20 & $\begin{array}{l}+0.10 \\
-0.10\end{array}$ & $\begin{array}{l}+0.26 \\
-0.19\end{array}$ & 0.99 & 0.83 \\
\hline \multicolumn{7}{|c|}{$250<Q^{2}<500 \mathrm{GeV}^{2}$} \\
\hline$-2.00--1.50$ & 8.65 & \pm 0.49 & $\begin{array}{l}+0.59 \\
-0.57\end{array}$ & $\begin{array}{l}+0.42 \\
-0.38\end{array}$ & 0.94 & 0.93 \\
\hline$-1.50--1.30$ & 23.4 & \pm 1.2 & ${ }_{-1.4}^{+1.5}$ & $\stackrel{+}{-1.3}_{1}^{+1.1}$ & 0.94 & 0.93 \\
\hline$-1.30--1.00$ & 16.18 & \pm 0.83 & $\begin{array}{l}+0.55 \\
-0.44\end{array}$ & $\begin{array}{l}+1.02 \\
{ }_{-0.78}\end{array}$ & 0.93 & 0.89 \\
\hline$-1.00--0.50$ & 1.77 & \pm 0.21 & $\begin{array}{l}+0.09 \\
{ }_{-0.09}\end{array}$ & $\begin{array}{l}+0.10 \\
{ }_{-0.11}\end{array}$ & 0.94 & 0.88 \\
\hline \multicolumn{7}{|c|}{$500<Q^{2}<1000 \mathrm{GeV}^{2}$} \\
\hline$-1.90--1.50$ & 4.32 & \pm 0.40 & $\begin{array}{l}+0.15 \\
-0.18\end{array}$ & $\begin{array}{l}+0.23 \\
-0.17\end{array}$ & 0.94 & 0.92 \\
\hline$-1.50--1.20$ & 17.50 & \pm 0.88 & $\begin{array}{l}+1.00 \\
-1.00\end{array}$ & ${ }_{-0.75}^{+0.72}$ & 0.94 & 0.94 \\
\hline$-1.20--0.90$ & 9.45 & \pm 0.63 & $\begin{array}{l}+0.90 \\
-0.90\end{array}$ & ${ }_{-0.35}^{+0.50}$ & 0.91 & 0.92 \\
\hline$-0.90--0.60$ & 1.48 & \pm 0.26 & $\begin{array}{l}+0.09 \\
-0.09 \\
\end{array}$ & $\begin{array}{l}+0.06 \\
-0.06 \\
\end{array}$ & 0.98 & 0.86 \\
\hline \multicolumn{7}{|c|}{$1000<Q^{2}<2000 \mathrm{GeV}^{2}$} \\
\hline$-1.70--1.40$ & 2.72 & \pm 0.37 & $\begin{array}{l}+0.33 \\
-0.33\end{array}$ & $\begin{array}{l}+0.06 \\
-0.12\end{array}$ & 0.93 & 0.94 \\
\hline$-1.40--1.20$ & 10.98 & \pm 0.86 & $\begin{array}{l}+1.23 \\
-1.23\end{array}$ & ${ }_{-0.01}^{+0.22}$ & 0.94 & 0.98 \\
\hline$-1.20--1.00$ & 10.37 & \pm 0.84 & $\begin{array}{l}+0.48 \\
-0.48\end{array}$ & $\begin{array}{l}+0.26 \\
-0.28\end{array}$ & 0.92 & 0.96 \\
\hline$-1.00--0.60$ & 2.17 & \pm 0.25 & $\begin{array}{l}+0.15 \\
-0.15 \\
\end{array}$ & $\begin{array}{l}+0.06 \\
-0.08 \\
\end{array}$ & 0.85 & 0.89 \\
\hline \multicolumn{7}{|c|}{$2000<Q^{2}<5000 \mathrm{GeV}^{2}$} \\
\hline$-1.60--1.20$ & 2.14 & \pm 0.26 & $\begin{array}{l}+0.14 \\
-0.14\end{array}$ & $\begin{array}{l}+0.01 \\
-0.02\end{array}$ & 0.92 & 0.99 \\
\hline$-1.20--1.00$ & 8.28 & \pm 0.80 & ${ }_{-0.54}^{+0.54}$ & ${ }_{-0.18}^{+0.14}$ & 1.00 & 0.96 \\
\hline$-1.00--0.60$ & 2.48 & \pm 0.30 & $\begin{array}{l}+0.16 \\
-0.16\end{array}$ & ${ }_{-0.04}^{+0.08}$ & 0.93 & 0.94 \\
\hline
\end{tabular}

Table 7: Dijet cross-sections $d \sigma / d \log _{10} \xi$ for jets of hadrons in the Breit frame selected with the longitudinally invariant $k_{T}$ cluster algorithm in different regions of $Q^{2}$. Other details as in the caption to Table 1. 


\begin{tabular}{|c|c|c|c|c|c|c|}
\hline $\begin{array}{c}E_{T, \mathrm{~B}}^{\text {jet }} \text { bin } \\
(\mathrm{GeV})\end{array}$ & $\begin{array}{l}d \sigma / d E_{T, \mathrm{~B}}^{\mathrm{jet}} \\
(\mathrm{pb} / \mathrm{GeV})\end{array}$ & $\delta_{\text {stat }}$ & $\delta_{\text {syst }}$ & $\delta_{\mathrm{ES}}$ & $C_{\mathrm{QED}}$ & $C_{\text {had }}$ \\
\hline \multicolumn{7}{|c|}{$125<Q^{2}<250 \mathrm{GeV}^{2}$} \\
\hline $8-10$ & 32.97 & 0.49 & $\begin{array}{l}+1.21 \\
-1.21\end{array}$ & $\begin{array}{l}+1.81 \\
-1.69\end{array}$ & 0.96 & 0.90 \\
\hline $10-14$ & 13.00 & 0.22 & $\begin{array}{l}+0.19 \\
{ }_{-0.19}\end{array}$ & $\begin{array}{l}+0.79 \\
{ }_{-0.75}\end{array}$ & 0.98 & 0.94 \\
\hline $14-18$ & 3.71 & 0.11 & $\begin{array}{l}+0.15 \\
+0.15\end{array}$ & ${ }_{-0.24}^{+0.28}$ & 0.97 & 0.94 \\
\hline $18-25$ & 0.835 & 0.037 & $\begin{array}{l}+0.013 \\
{ }_{-0.012}\end{array}$ & $\begin{array}{l}+0.051 \\
{ }_{-0.056}\end{array}$ & 0.94 & 0.93 \\
\hline $25-100$ & 0.0160 & 0.0014 & $\begin{array}{l}+0.0027 \\
-0.0027\end{array}$ & $\begin{array}{l}+0.0010 \\
{ }_{-0.0011}\end{array}$ & 0.97 & 0.86 \\
\hline \multicolumn{7}{|c|}{$250<Q^{2}<500 \mathrm{GeV}^{2}$} \\
\hline $8-10$ & 18.40 & 0.38 & $\begin{array}{l}+0.74 \\
-0.74\end{array}$ & $\begin{array}{l}+0.68 \\
{ }_{-0.60}\end{array}$ & 0.94 & 0.92 \\
\hline $10-14$ & 8.74 & 0.19 & ${ }_{-0.30}^{+0.30}$ & $\begin{array}{l}+0.33 \\
-0.35\end{array}$ & 0.96 & 0.95 \\
\hline $14-18$ & 3.30 & 0.11 & $\begin{array}{l}+0.15 \\
-0.15\end{array}$ & $\begin{array}{l}+0.18 \\
-0.14\end{array}$ & 0.96 & 0.97 \\
\hline $18-25$ & 0.889 & 0.042 & $\begin{array}{l}+0.041 \\
{ }_{-0.041}\end{array}$ & $\begin{array}{l}+0.052 \\
{ }_{-0.057}\end{array}$ & 0.92 & 0.97 \\
\hline $25-100$ & 0.0242 & 0.0020 & $\begin{array}{l}+0.0005 \\
{ }_{-0.0005}\end{array}$ & $\begin{array}{l}+0.0012 \\
{ }_{-0.0011}\end{array}$ & 0.95 & 0.91 \\
\hline \multicolumn{7}{|c|}{$500<Q^{2}<1000 \mathrm{GeV}^{2}$} \\
\hline $8-10$ & 8.79 & 0.26 & $\begin{array}{l}+0.34 \\
-0.34\end{array}$ & $\begin{array}{l}+0.26 \\
{ }_{-0.15}^{0}\end{array}$ & 0.96 & 0.91 \\
\hline $10-14$ & 4.69 & 0.14 & $\begin{array}{l}+0.19 \\
{ }_{-0.19}\end{array}$ & $\begin{array}{l}+0.11 \\
{ }_{-0.13}\end{array}$ & 0.94 & 0.95 \\
\hline $14-18$ & 2.239 & 0.093 & $\begin{array}{l}+0.137 \\
{ }_{-0.137}\end{array}$ & $\begin{array}{l}+0.091 \\
{ }_{-0.074}\end{array}$ & 0.93 & 0.98 \\
\hline $18-25$ & 0.701 & 0.039 & $\begin{array}{l}+0.051 \\
-0.051\end{array}$ & $\begin{array}{l}+0.026 \\
-0.026\end{array}$ & 0.96 & 0.99 \\
\hline $25-100$ & 0.0335 & 0.0027 & $\begin{array}{l}+0.0018 \\
{ }_{-0.0018}\end{array}$ & $\begin{array}{l}+0.0019 \\
{ }_{-0.0019}^{+}\end{array}$ & 0.96 & 0.97 \\
\hline \multicolumn{7}{|c|}{$1000<Q^{2}<2000 \mathrm{GeV}^{2}$} \\
\hline $8-10$ & 3.30 & 0.16 & $\begin{array}{l}+0.14 \\
-0.14\end{array}$ & $\begin{array}{l}+0.09 \\
+0.08\end{array}$ & 0.93 & 0.93 \\
\hline $10-14$ & 1.985 & 0.091 & $\begin{array}{l}+0.077 \\
{ }_{-0.077}\end{array}$ & ${ }_{-0.037}^{+0.017}$ & 0.91 & 0.95 \\
\hline $14-18$ & 1.115 & 0.069 & $\begin{array}{l}+0.056 \\
{ }_{-0.056}\end{array}$ & $\begin{array}{l}+0.034 \\
{ }_{-0.008}\end{array}$ & 0.98 & 0.99 \\
\hline $18-25$ & 0.492 & 0.034 & $\begin{array}{l}+0.039 \\
{ }_{-0}^{+0.039}\end{array}$ & $\begin{array}{l}{ }_{-0.009}^{+0.009} \\
-0.018\end{array}$ & 0.93 & 0.99 \\
\hline $25-100$ & 0.0263 & 0.0026 & $\begin{array}{l}+0.0043 \\
{ }_{-0}^{+0.0043}\end{array}$ & $\begin{array}{l}+0.0020 \\
{ }_{-0.0013}\end{array}$ & 1.00 & 1.00 \\
\hline \multicolumn{7}{|c|}{$2000<Q^{2}<5000 \mathrm{GeV}^{2}$} \\
\hline $8-10$ & 1.292 & 0.095 & $\begin{array}{l}+0.120 \\
{ }_{-0.120}^{+}\end{array}$ & $\begin{array}{l}+0.033 \\
{ }_{-0.022}\end{array}$ & 0.92 & 0.90 \\
\hline $10-14$ & 0.858 & 0.060 & $\begin{array}{l}+0.024 \\
-0.024\end{array}$ & ${ }_{-0}^{+0}$ & 0.90 & 0.93 \\
\hline $14-18$ & 0.612 & 0.052 & $\begin{array}{l}+0.070 \\
{ }_{-0.070}\end{array}$ & $\begin{array}{l}+0.023 \\
{ }_{-0.017}\end{array}$ & 1.02 & 1.00 \\
\hline $18-25$ & 0.242 & 0.024 & $\begin{array}{l}-0.028 \\
{ }_{-0.028}^{+0.028}\end{array}$ & $\begin{array}{l}-0.009 \\
+0.006\end{array}$ & 0.96 & 1.00 \\
\hline $25-100$ & 0.0185 & 0.0021 & $\begin{array}{l}+0.0023 \\
{ }_{-0.0023}\end{array}$ & $\begin{array}{l}+0.0004 \\
-0.0005\end{array}$ & 0.91 & 0.99 \\
\hline \multicolumn{7}{|c|}{$5000<Q^{2}<100000 \mathrm{GeV}^{2}$} \\
\hline $8-10$ & 0.225 & 0.037 & $\begin{array}{l}+0.091 \\
{ }_{-0.091}^{+}\end{array}$ & $\begin{array}{l}+0.011 \\
{ }_{-0.006}\end{array}$ & 0.99 & 0.93 \\
\hline $10-14$ & 0.267 & 0.037 & & & 0.96 & 0.93 \\
\hline $14-18$ & 0.122 & 0.024 & $\begin{array}{l}+0.017 \\
{ }_{-0.017}\end{array}$ & $\begin{array}{l}+0.003 \\
{ }_{-0.005}\end{array}$ & 0.97 & 0.98 \\
\hline $18-25$ & 0.070 & 0.013 & ${ }_{-0}^{+0}$ & $\begin{array}{l}+0.001 \\
{ }_{-0.000}\end{array}$ & 0.98 & 0.99 \\
\hline $25-100$ & 0.0114 & 0.0022 & $\begin{array}{l}+0.0042 \\
{ }_{-0.0042}\end{array}$ & $\begin{array}{l}+0.0003 \\
{ }_{-0.0001}\end{array}$ & 0.99 & 1.00 \\
\hline
\end{tabular}

Table 8: Inclusive jet cross-sections $d \sigma / d E_{T, \mathrm{~B}}^{\mathrm{jet}}$ for jets of hadrons in the Breit frame selected with the longitudinally invariant $k_{T}$ cluster algorithm in different regions of $Q^{2}$. Other details as in the caption to Table 1. 


\section{ZEUS}
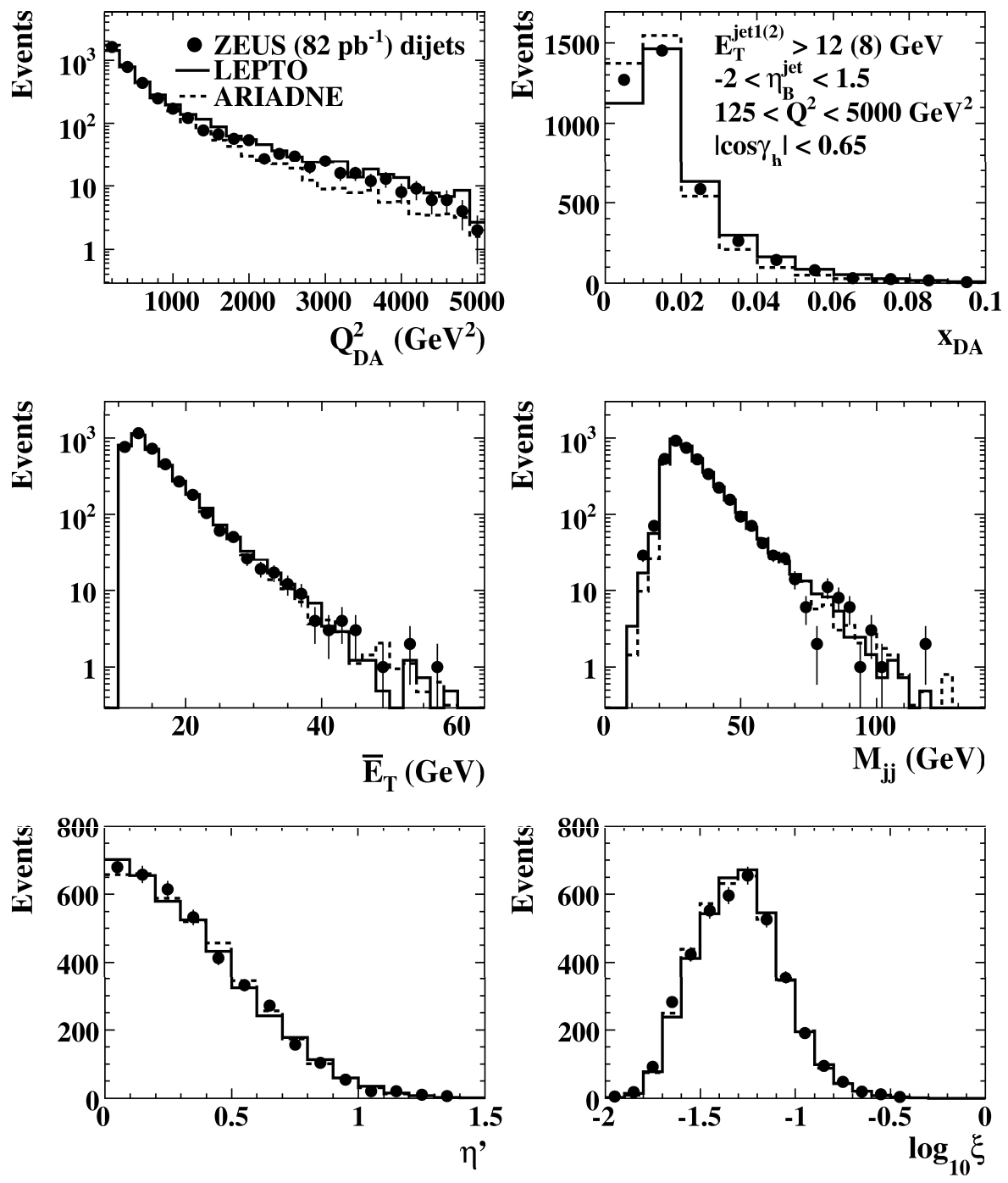

Figure 1: Uncorrected data distributions for dijet production with $E_{T, \mathrm{~B}}^{\mathrm{jet} 1}>12 \mathrm{GeV}$, $E_{T, \mathrm{~B}}^{\mathrm{jet} 2}>8 \mathrm{GeV}$ and $-2<\eta_{\mathrm{B}}^{\mathrm{jet}}<1.5$ in the kinematic range given by $\left|\cos \gamma_{h}\right|<0.65$ and $125<Q^{2}<5000 \mathrm{GeV}^{2}$ (dots). For comparison, the predictions of the ARIADNE (dashed histograms) and LEPTO (solid histograms) MC models are also included. 


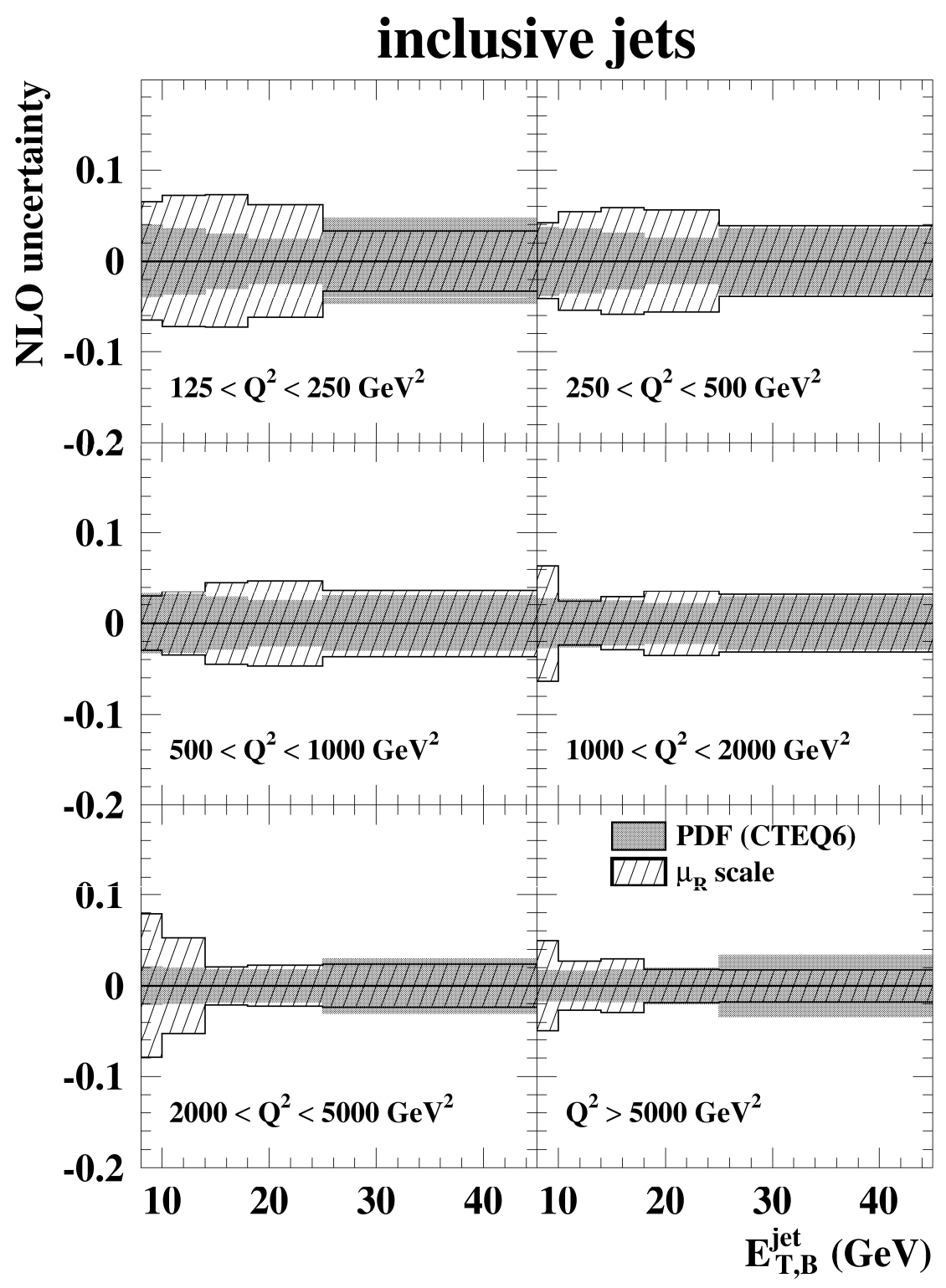

Figure 2: Overview of theoretical uncertainties for the inclusive-jet analysis as functions of $E_{T, \mathrm{~B}}^{\mathrm{jet}}$ in bins of $Q^{2}$ for $\left|\cos \gamma_{h}\right|<0.65$ and $-2<\eta_{\mathrm{B}}^{\text {jet }}<1.5$. Shown are the relative uncertainties induced by the variation of the renormalisation scale $\mu_{R}$ (hatched area) and by the uncertainties on the proton PDFs (shaded area). 


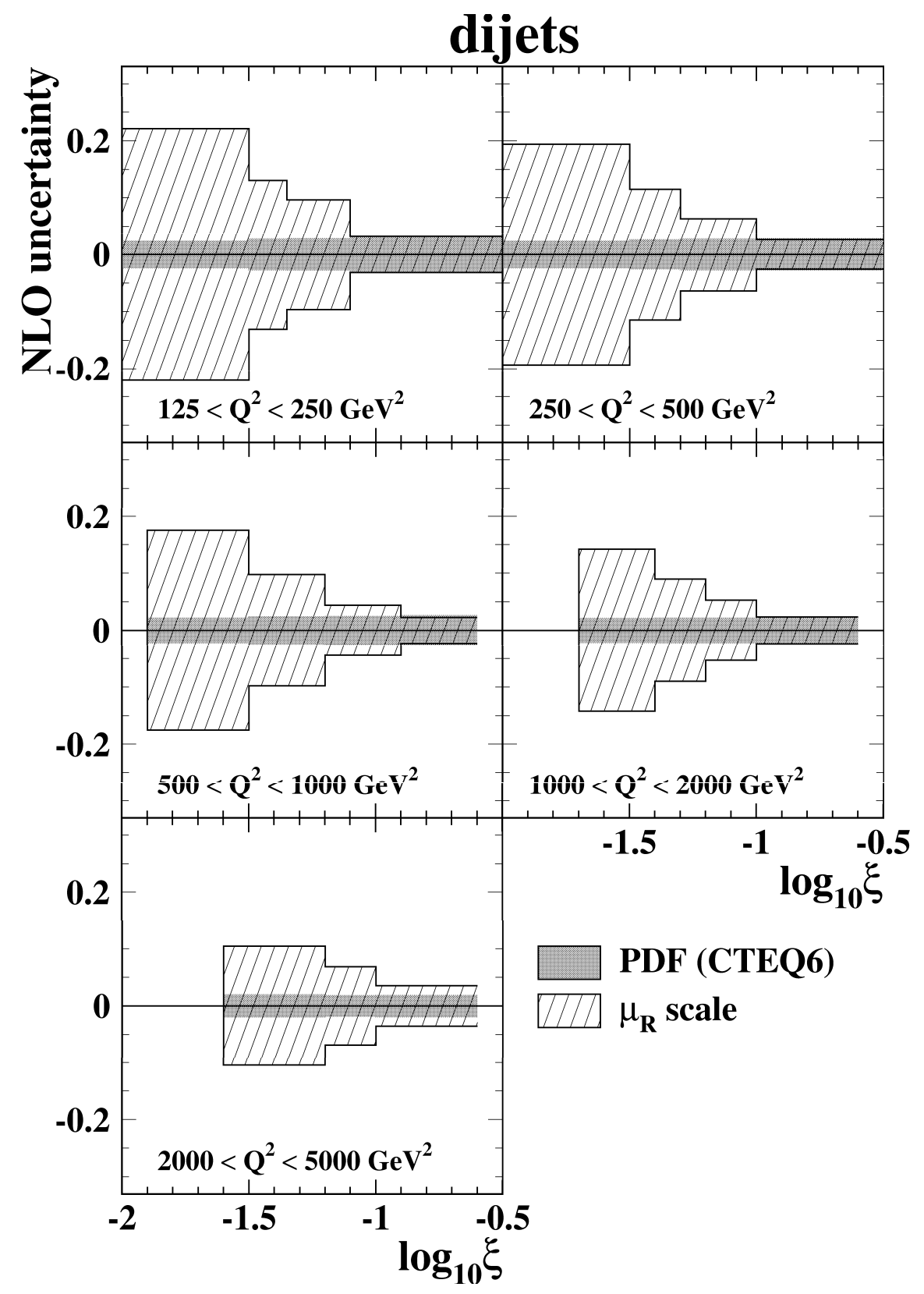

Figure 3: Overview of theoretical uncertainties for the dijet analysis as functions of $\log _{10} \xi$ in bins of $Q^{2}$ for $\left|\cos \gamma_{h}\right|<0.65, E_{T, \mathrm{~B}}^{\text {jet }}>12 \mathrm{GeV}, E_{T, \mathrm{~B}}^{\text {jet }}>8 \mathrm{GeV}$ and $-2<\eta_{\mathrm{B}}^{\mathrm{jet}}<1.5$. Other details as in the caption to Fig. 2. 


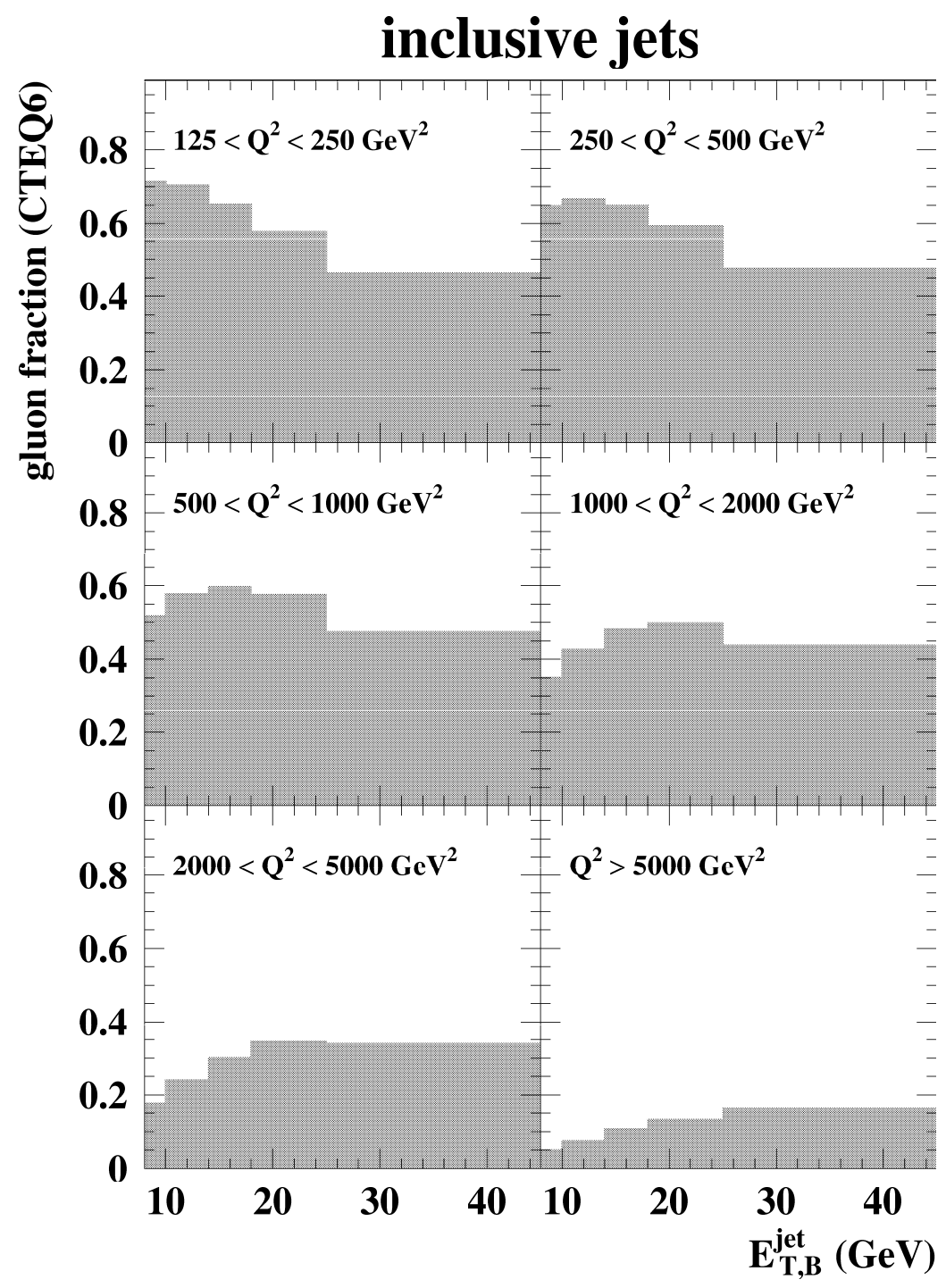

Figure 4: $\quad$ NLO QCD predictions of the gluon-induced fraction of the inclusivejet cross sections $d \sigma / d E_{T, \mathrm{~B}}^{\mathrm{jet}}$ as functions of $E_{T, B}^{\text {jet }}$ in different regions of $Q^{2}$ for $\left|\cos \gamma_{h}\right|<0.65$ and $-2<\eta_{\mathrm{B}}^{\text {jet }}<1.5$ (shaded histograms). The CTEQ6 parameterisations of the proton PDFs were used. 


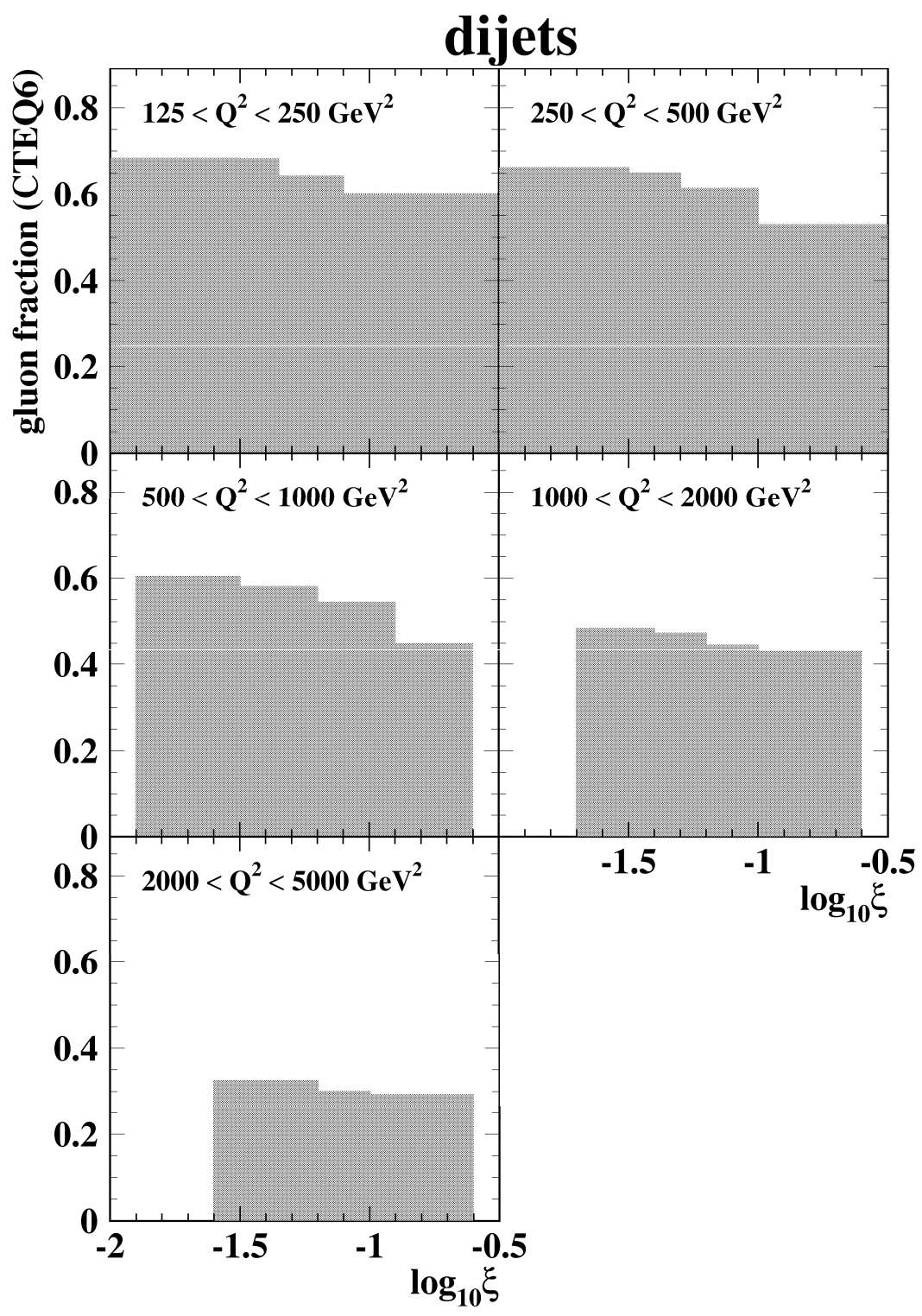

Figure 5: $\quad N L O Q C D$ predictions of the gluon-induced fraction of the dijet cross-sections $d \sigma / d \log _{10} \xi$ as functions of $\log _{10} \xi$ in different regions of $Q^{2}$ for $\left|\cos \gamma_{h}\right|<0.65, E_{T, \mathrm{~B}}^{\text {jet } 1}>12 \mathrm{GeV}, E_{T, \mathrm{~B}}^{\text {jet } 2}>8 \mathrm{GeV}$ and $-2<\eta_{\mathrm{B}}^{\text {jet }}<1.5$. Other details as in the caption to Fig. 4. 


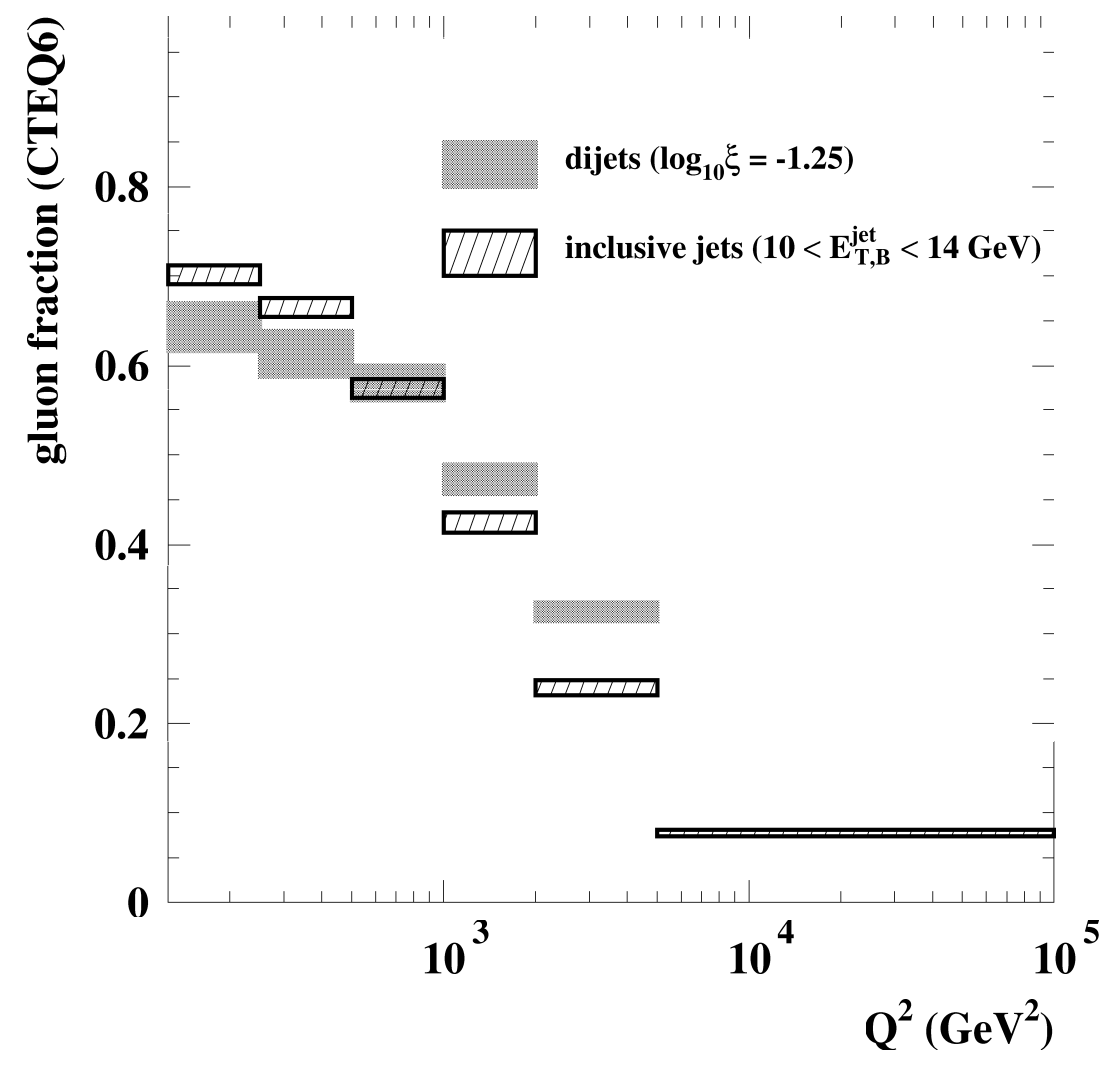

Figure 6: $\quad N L O$ QCD predictions of the gluon-induced fraction of the dijet (inclusive-jet) cross-section $d \sigma / d \log \xi\left(d \sigma / d E_{T, \mathrm{~B}}^{\mathrm{jet}}\right)$ as a function of $Q^{2}$ for $\left|\cos \gamma_{h}\right|<0.65$ and $-2<\eta_{\mathrm{B}}^{\text {jet }}<1.5$ and for $\log _{10} \xi=-1.25\left(10<E_{T, \mathrm{~B}}^{\mathrm{jet}}<14 \mathrm{GeV}\right)$; the uncertainty on the gluon-induced fraction due to those on the proton PDFs is represented by the shaded (hatched) area. The CTEQ6 parameterisations of the proton PDFs were used. 


\section{ZEUS}
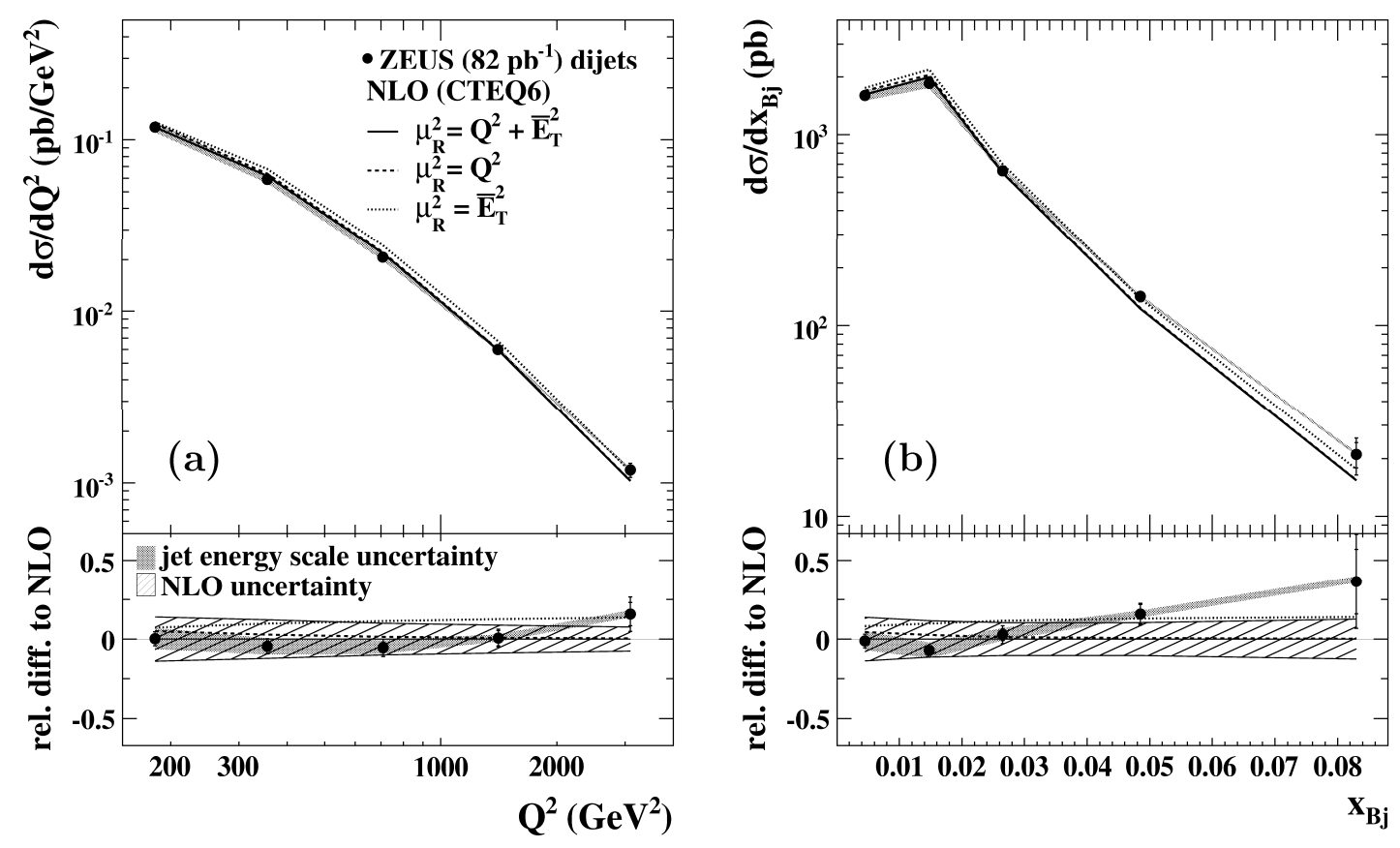

Figure 7: The measured differential cross-sections (a) $d \sigma / d Q^{2}$ and (b) $d \sigma / d x_{\mathrm{Bi}}$ for dijet production with $E_{T, \mathrm{~B}}^{\mathrm{jet} 1}>12 \mathrm{GeV}, E_{T, \mathrm{~B}}^{\mathrm{jet} 2}>8 \mathrm{GeV}$ and $-2<\eta_{\mathrm{B}}^{\text {jet }}<1.5$ (dots), in the kinematic range given by $\left|\cos \gamma_{h}\right|<0.65$ and $125<Q^{2}<5000 \mathrm{GeV}^{2}$. The inner error bars represent the statistical uncertainty. The outer error bars show the statistical and systematic uncertainties, not associated with the uncertainty in the absolute energy scale of the jets, added in quadrature. The shaded bands display the uncertainties due to the absolute energy scale of the jets. The NLO QCD calculations with $\mu_{R}^{2}=Q^{2}+\bar{E}_{T}^{2}$ (solid lines), $\mu_{R}^{2}=Q^{2}$ (dashed lines) and $\mu_{R}^{2}=\bar{E}_{T}^{2}$ (dash-dotted lines), corrected for hadronisation effects and using the CTEQ6 parameterisations of the proton PDFs, are also shown. The lower parts of the figures show the relative differences with respect to the NLO QCD calculations with $\mu_{R}^{2}=Q^{2}+\bar{E}_{T}^{2}$ : measurements (dots), NLO QCD calculations with $\mu_{R}^{2}=Q^{2}$ (dashed lines) and with $\mu_{R}^{2}=\bar{E}_{T}^{2}$ (dotted lines); the hatched bands display the total theoretical uncertainty. 


\section{ZEUS}
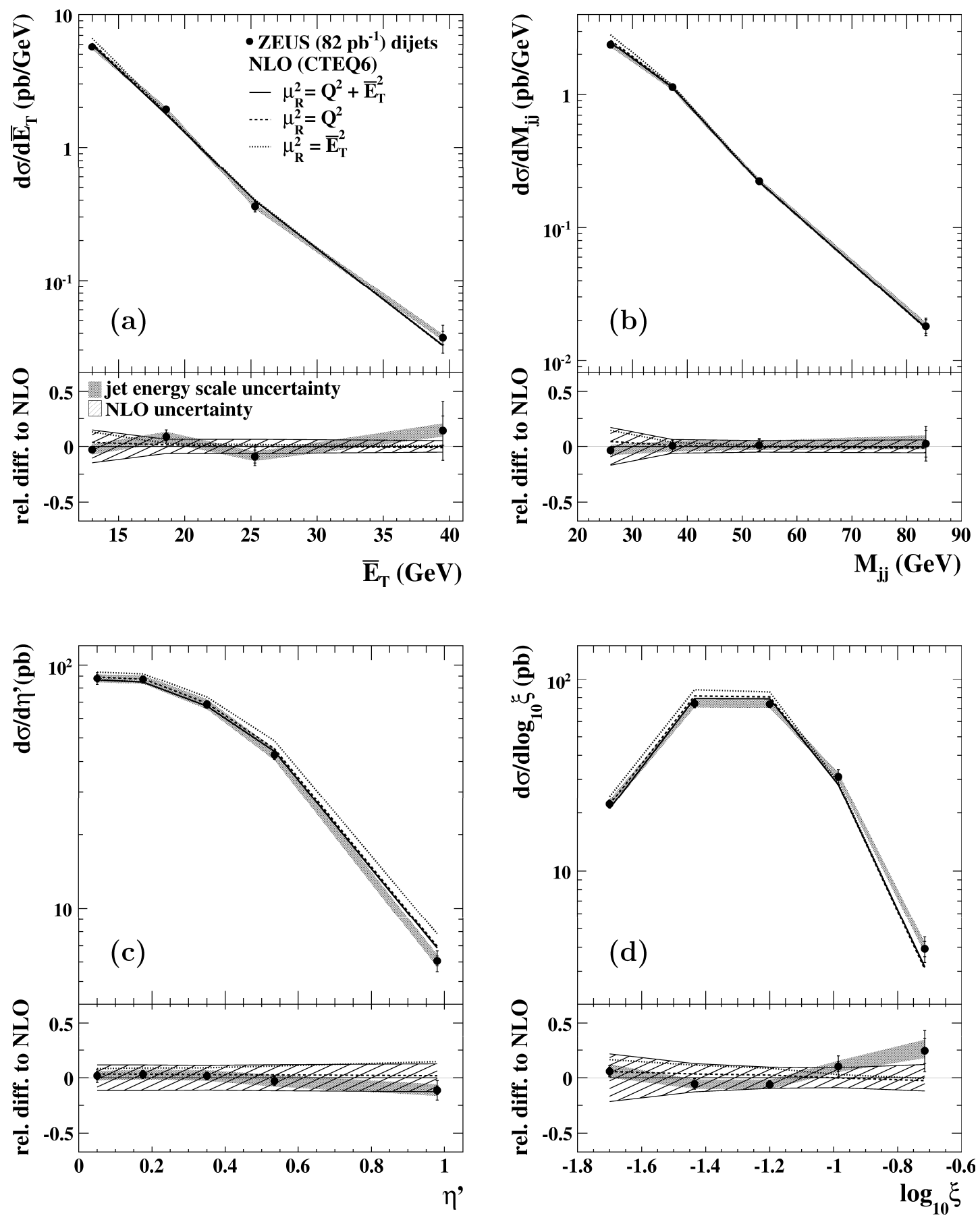

Figure 8: The measured differential cross-sections (a) $d \sigma / d \bar{E}_{T}$, (b) $d \sigma / d M_{\mathrm{jj}}$, (c) $d \sigma / d \eta^{\prime}$ and $(d) d \sigma / d \log _{10} \xi$ for dijet production with $E_{T, \mathrm{~B}}^{\mathrm{jet}}>12 \mathrm{GeV}, E_{T, \mathrm{~B}}^{\mathrm{jet} 2}>8 \mathrm{GeV}$ and $-2<\eta_{\mathrm{B}}^{\mathrm{jet}}<1.5$ (dots), in the kinematic range given by $\left|\cos \gamma_{h}\right|<0.65$ and $125<Q^{2}<5000 \mathrm{GeV}^{2}$. Other details as in the caption to Fig. $\%$. 


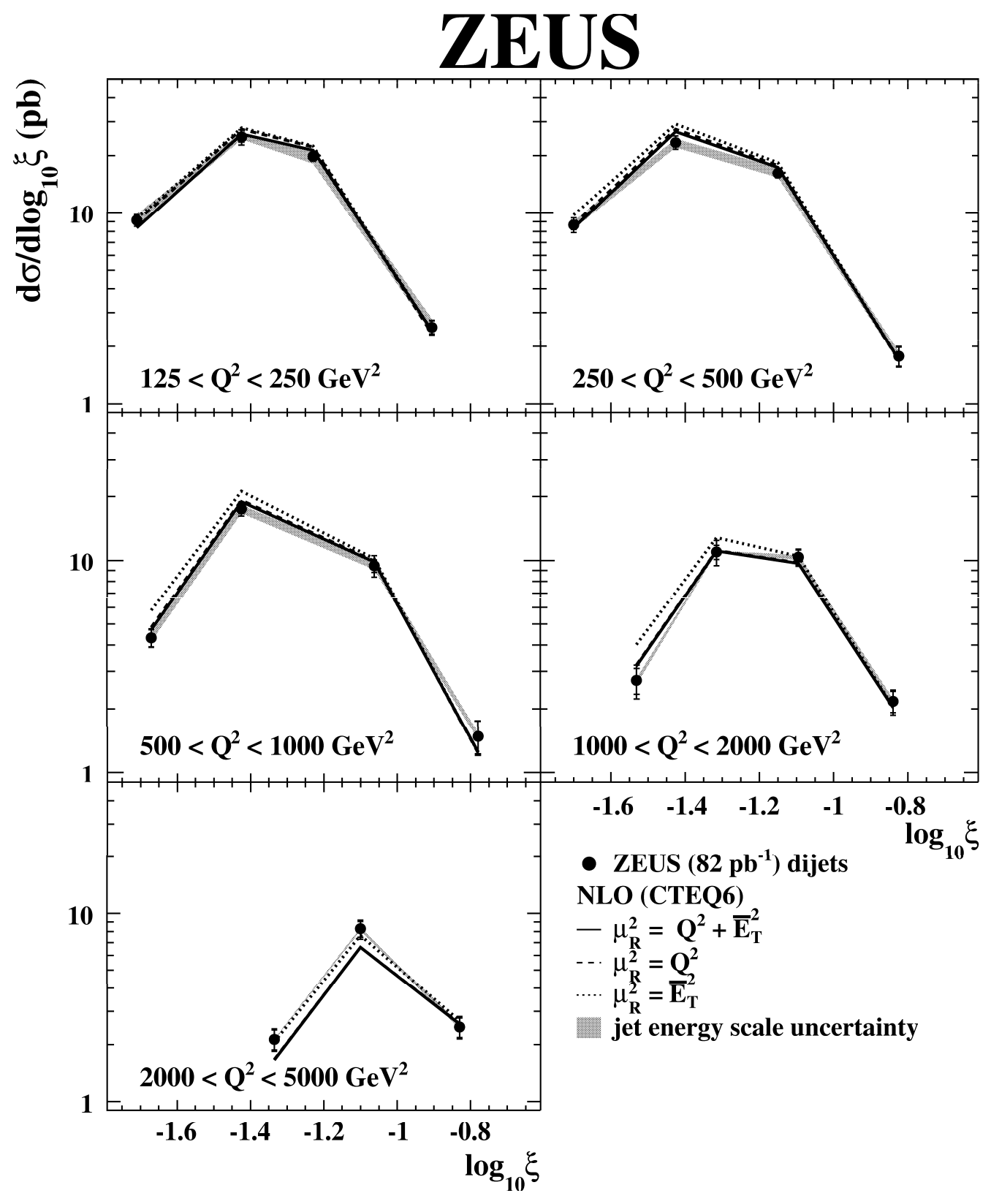

Figure 9: The measured differential cross-section $d \sigma / d \log _{10} \xi$ for dijet production with $E_{T, \mathrm{~B}}^{\mathrm{jet} 1}>12 \mathrm{GeV}, E_{T, \mathrm{~B}}^{\mathrm{jet} 2}>8 \mathrm{GeV}$ and $-2<\eta_{\mathrm{B}}^{\mathrm{jet}}<1.5$ in different regions of $Q^{2}$ (dots). Other details as in the caption to Fig. 7. 


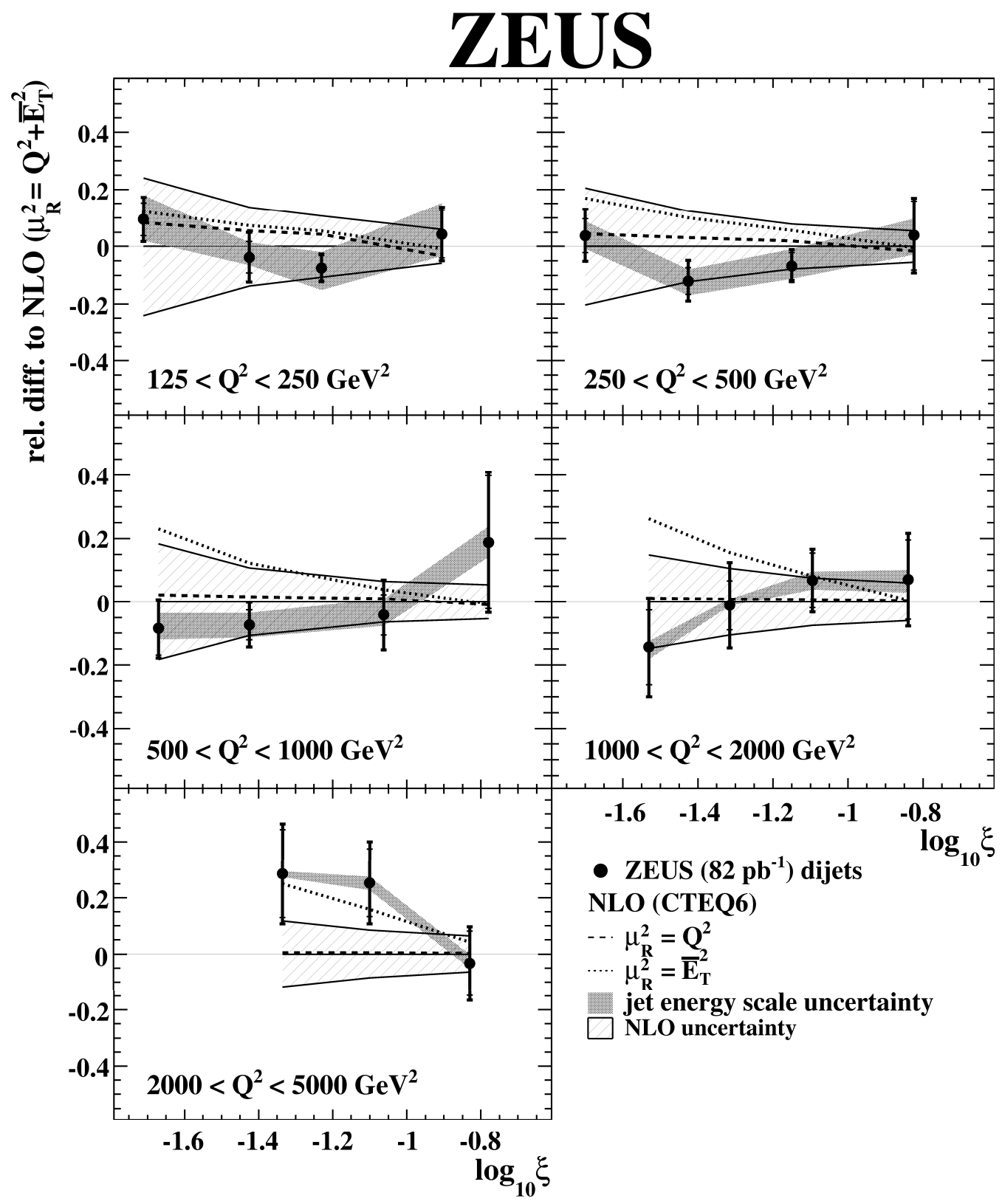

Figure 10: Relative differences between the measured differential cross-sections $d \sigma / d \log _{10} \xi$ presented in Fig. 9 and the NLO QCD calculations with $\mu_{R}^{2}=Q^{2}+\bar{E}_{T}^{2}$ (dots). The relative differences between the NLO QCD calculations with $\mu_{R}^{2}=Q^{2}$ $\left(\mu_{R}^{2}=\bar{E}_{T}^{2}\right)$ and those with $\mu_{R}^{2}=Q^{2}+\bar{E}_{T}^{2}$ are also shown as dashed (dotted) lines. Other details as in the caption to Fig. $\%$. 


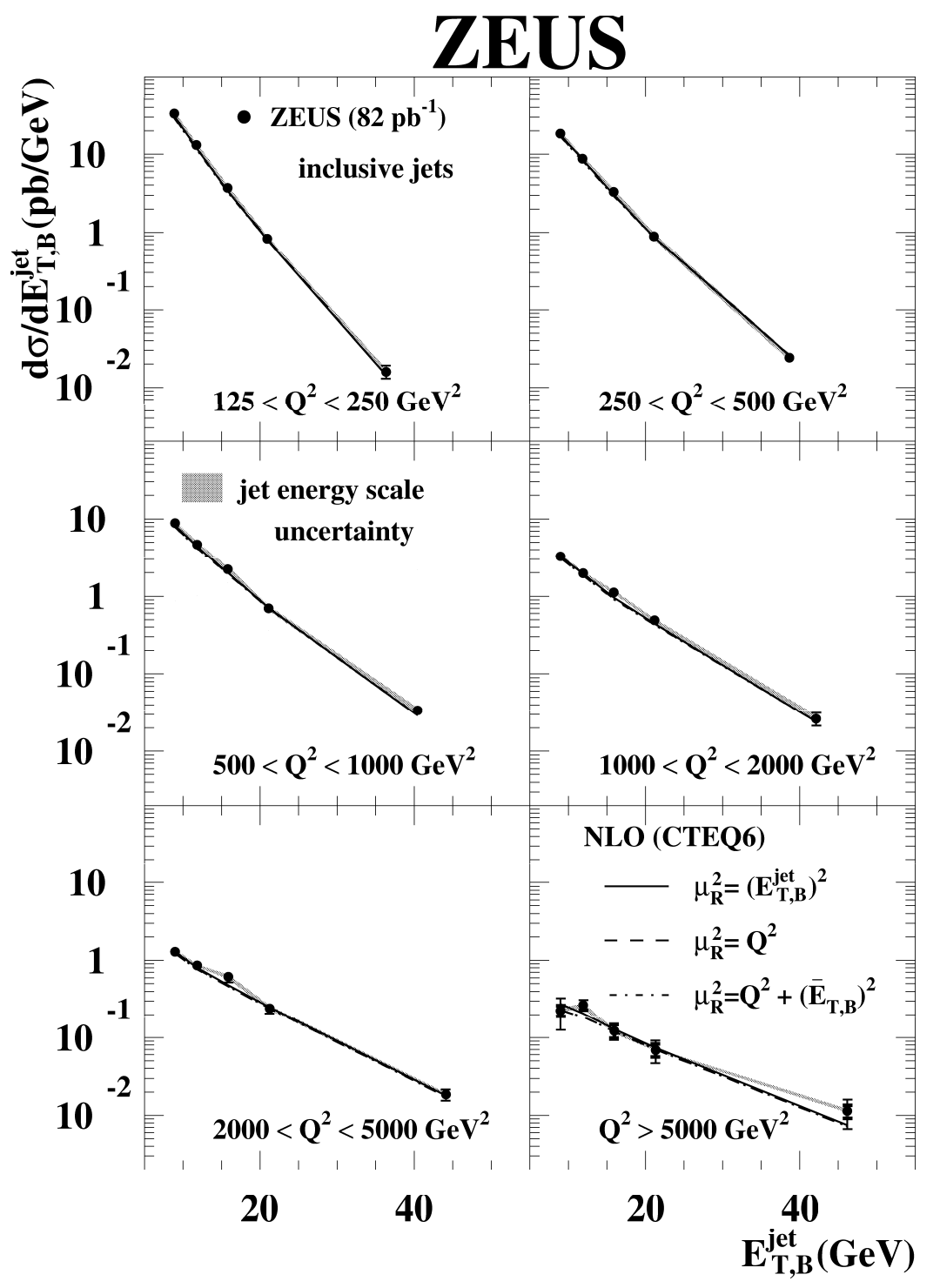

Figure 11: The measured differential cross-section $d \sigma / d E_{T, \mathrm{~B}}^{\mathrm{jet}}$ for inclusive-jet production with $E_{T, \mathrm{~B}}^{\mathrm{jet}}>8 \mathrm{GeV}$ and $-2<\eta_{\mathrm{B}}^{\mathrm{jet}}<1.5$ in different regions of $Q^{2}$ (dots). The NLO QCD calculations with $\mu_{R}^{2}=\left(E_{T, \mathrm{~B}}^{\mathrm{jet}}\right)^{2}$ (solid lines), $\mu_{R}^{2}=Q^{2}$ (dashed lines) and $\mu_{R}^{2}=Q^{2}+\bar{E}_{T, \mathrm{~B}}^{2}$ (dash-dotted lines), corrected for hadronisation and $Z^{0}$ effects and using the CTEQ6 parameterisations of the proton PDFs, are also shown. Other details as in the caption to Fig. 7. 


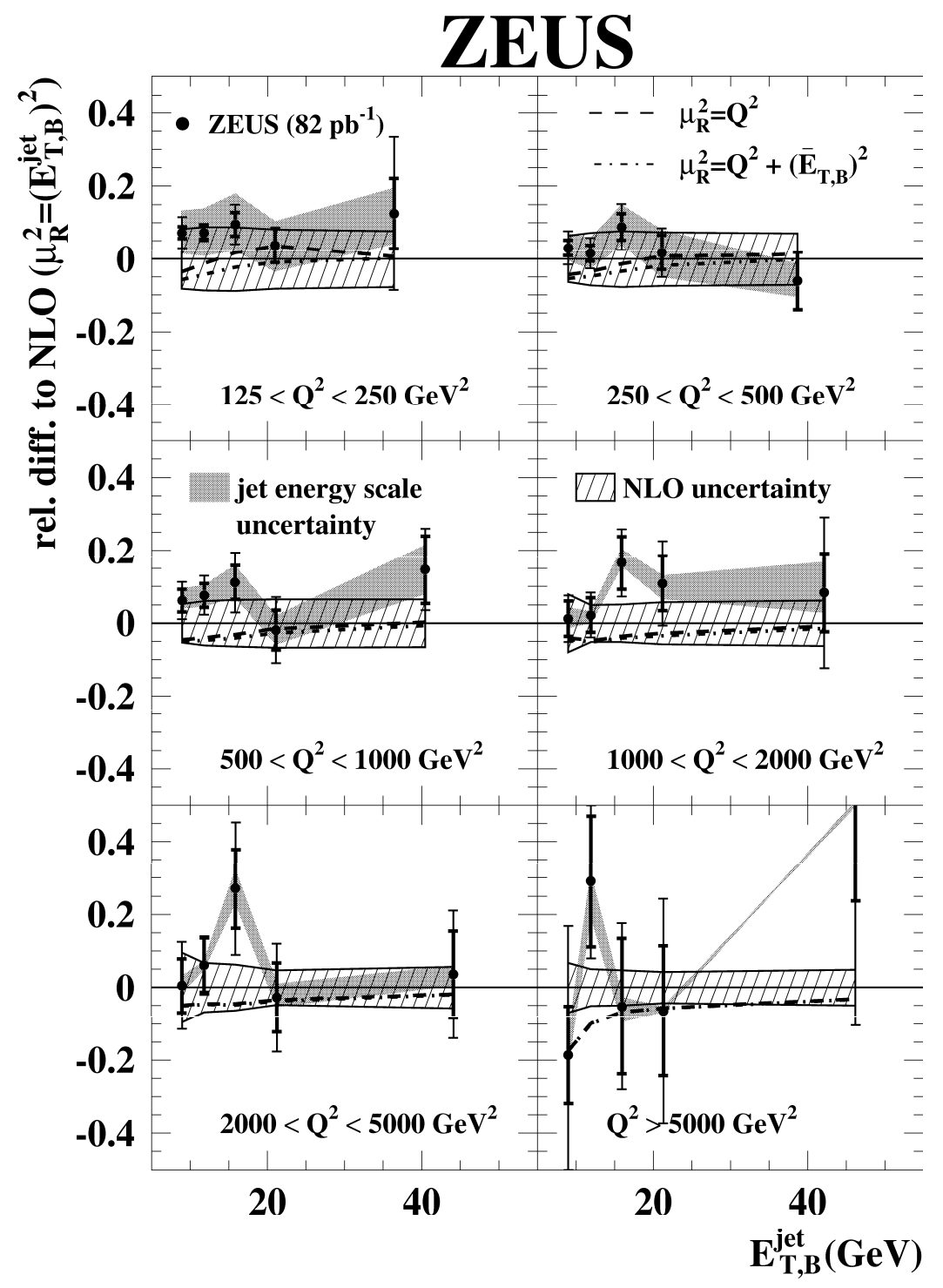

Figure 12: Relative differences between the measured differential cross-sections $d \sigma / d E_{T, \mathrm{~B}}^{\mathrm{jet}}$ presented in Fig. 11 and the $N L O Q C D$ calculations with $\mu_{R}^{2}=\left(E_{T, B}^{\text {jet }}\right)^{2}$ (dots). The relative differences between the NLO QCD calculations with $\mu_{R}^{2}=Q^{2}$ $\left(\mu_{R}^{2}=Q^{2}+\bar{E}_{T, \mathrm{~B}}^{2}\right)$ and those with $\mu_{R}^{2}=\left(E_{T, B}^{\text {jet }}\right)^{2}$ are also shown as dashed (dashdotted) lines. Other details as in the caption to Fig. $\%$. 